\title{
Review Article \\ Visualization Methods of 3D Plant Models: A Systematic Mapping Study
}

\author{
Shilin Di $(\mathbb{D}$, Lingzhi Cao $\mathbb{D}$, and Yongqi Ge $\mathbb{1}$ \\ School of Information Engineering, Ningxia University, Yinchuan 750021, China \\ Correspondence should be addressed to Yongqi Ge; geyongqi@nxu.edu.cn
}

Received 7 June 2021; Revised 11 August 2021; Accepted 30 August 2021; Published 9 September 2021

Academic Editor: Hector E. Nistazakis

Copyright (C) 2021 Shilin Di et al. This is an open access article distributed under the Creative Commons Attribution License, which permits unrestricted use, distribution, and reproduction in any medium, provided the original work is properly cited.

\begin{abstract}
Three-dimensional plant model visualization (3D-PMV) is based on plant biology and the plant structure construction model for virtual simulation three-dimensional display, reflecting plant structure characteristics and demonstrating the growth process. In this work, we used the method of systematic mapping study (SMS) to screen the relevant literature in order to explore and analyze the methods and goals of virtual plant visualization research and to provide assistance in future literature reviews. To this end, we conducted extensive searches to identify articles related to plant model visualization technology. We searched for papers from July 2010 to November 2020 from four mainstream databases, namely, ACM, IEEE Xplore, EI, and Web of Science, and found more than 2,900 papers on 3D-PMV. Finally, we selected 60 qualified papers. We mainly followed the SMS method to classify papers by answering seven questions, mainly extracting data for analysis on research types, research goals, model construction methods, visual model categories, number of publications, tools, and methods. The results show that solution proposals account for the largest proportion of research types, accounting for $75 \%$ of the total number of papers. This shows that the focus is on the improvement of original methods or the proposal of new methods in this field; research goals of research plant phenotypic characteristics account for the total 35\%; $28 \%$ of the research objectives focus on showing the reflection of plant growth process, indicating that the research objective trends in this research field regard plant phenotypic characteristics and visualization of the growth process; static type model visualization also accounts for $83 \%$ of the total, indicating that the related research on visualization in this field is mostly static typing. Therefore, our work not only analyzes the challenges faced by 3D-PVM and the research directions that need more attention in the future but also provides some suggestions for researchers to find suitable research directions in this field. As the future direction of development, researchers need to pay more attention to the study of dynamic visualization methods of plant growth. We believe that for future research, it is indispensable to provide 3D-PMV with research methods, research goals, visualization model types, development, and other tools. This article analyzes the results of the extracted data based on the SMS method and makes an important contribution to the researcher's extensive understanding of the current status of 3D-PMV and the potential future research opportunities in the field.
\end{abstract}

\section{Introduction}

With the rapid development of computer virtual plant technology and agricultural information technology, plant three-dimensional (3D) visualization is playing an increasingly important role in observing plant growth dynamics, studying plant physiological structure, and planting management. Due to the complexity of the morphological structure of different plants and the diversity of morphological characteristics of the same plant at different growth stages, there are great differences in the $3 \mathrm{D}$ visualization methods of constructing plants. For example, the research of plant 3D visualization technology mainly focuses on plant mathematical model, computer tomography, and plant image feature extraction. The summary of the differences between these methods is based on systematic research in the research field.

Only after in-depth research and analysis of a particular research field can we summarize the current status of research in this field. At present, some methods of literature research such as systematic mapping study (SMS) or systematic review (SR) have been proposed. SMS is a rough overview of a certain field. In SMS research, more articles in a certain field can be considered, and no detailed evaluation 
is necessary. For example, the number and classification of research works published in this field are usually presented graphically. A SMS can help reviewers find research topics in specific fields in a wide range and can also help them to better track research-related fields.

Compared with SMS, SR conducts an in-depth analysis of the research focus of the limited search field and describes their methods and results. Therefore, SR is an extension of SMS. In this work, the focus of using SMS is to provide an overview of $3 \mathrm{D}$ plant model visualization methods, determine the research evidence for this topic, and present the main quantitative results $[1,2]$. We conducted this work to answer seven different research questions about 3D-PMV. Therefore, before using the SMS method, we read many papers that borrowed from the method and collected relevant research materials (see, e.g., [3-8]). According to the method of plant model construction, visual model category, research type, research objective, plant type, tools, methods, and publishing site, the research is classified, and the required data are collected by reading the title, abstract, keywords, and full text. Considering that $3 \mathrm{D}-\mathrm{PMV}$ is of great significance for observing plant growth dynamics, studying plant physiological structure, and planting management, it illustrates the importance of systematic analysis and provides researchers with a comprehensive understanding of values in this field over the past ten years. Therefore, this work fills this gap for the current literature review.

In this paper, we focus on exploring and analyzing the reality of the 3D-PMV field. The subsequent parts of this paper are arranged as follows. The SMS is described in Section 2. In Section 3, we introduce the screening results and answer the research questions. Then, in Section 4 , the summary and challenges of the 3D-PMV domain system are presented. Finally, a summary of our work and future prospects are given in Section 5.

\section{The Systematic Mapping Processes}

In this section, we follow the five steps of the systematic mapping process provided by Feldt et al. to complete the system map of this research field $[1,2]$. As shown in Figure 1 [9], SMS completion is divided into five steps as a whole. First, we need to define the research question and determine the scope of a research question paper. Second, a preliminary research search is used to find all papers in this range. Third, by screening the included and excluded papers, the relevant papers in the field of the research question are identified. Then, the abstracts of related papers are read, the keywords and research concepts that can explain the contribution of the papers are identified, and then they are combined after sorting them together, which will help to form an understanding of the research theme and contributions and thus form the classification of the papers. Finally, according to the above classification scheme, the papers related to the field of the research question selected in the scheme are classified, and data are extracted. These data are used to analyze all categories, and finally the system map is displayed by drawing.
2.1. Research Question (RQ). According to SMS, the main objective is to identify research types, research objectives, modeling approaches, visual dimensions, visual model categories, tools, methods, and publications relevant to the 3D-PMV field since 2010. The following is a detailed description of these research questions:

(i) RQ1: what types of research have been conducted in the field of 3D-PMV over a period of time and how has it developed?

Basic Principle. The understanding of the current status of popular research types in the set research field helps researchers to understand the research trends and future development forms of the field, and at the same time, it leaves investigators thinking about the future development direction of the field. Popular research trends in the field of 3D plant visualization can be identified through SMS, which is the core of our research.

(ii) RQ2: what are the research goals?

Basic Principle. Since the goals of each paper to achieve 3D-PMV are not exactly the same, the selected studies are grouped according to the different research goals of the selected papers. We provide data analysis based on the actual classification results of the research objectives.

(iii) RQ3: which studies in 3D-PMV use traditional model building methods?

Basic Principle. In the research of related issues, many researchers realize that the initial model of 3D-PMV belongs to the traditional way of constructing the model. Our focus is to identify the practice of most people in this related field.

(iv) RQ4: which dimension realizes the visualization of the plant model?

Basic Principle. Our focus is to identify the currently popular visualization dimensions for plant model visualization methods. The reason for setting this question is that we have excluded two-dimensional visualization that does not meet the research theme of this article and discovered the progress of the visualization dimension method in the process of paper screening.

(v) RQ5: what are the categories of 3D plant visualization models?

Basic Principle. Our purpose is to classify according to the different functions realized by the visualization model. This question will answer different visualization models to realize the function goals and explain the research trend in the field of visualization models.

(vi) RQ6: how many method papers and tool papers are proposed in these related studies?

Basic Principle. Our goal is to identify the tools and methods that researchers can use in these studies. 

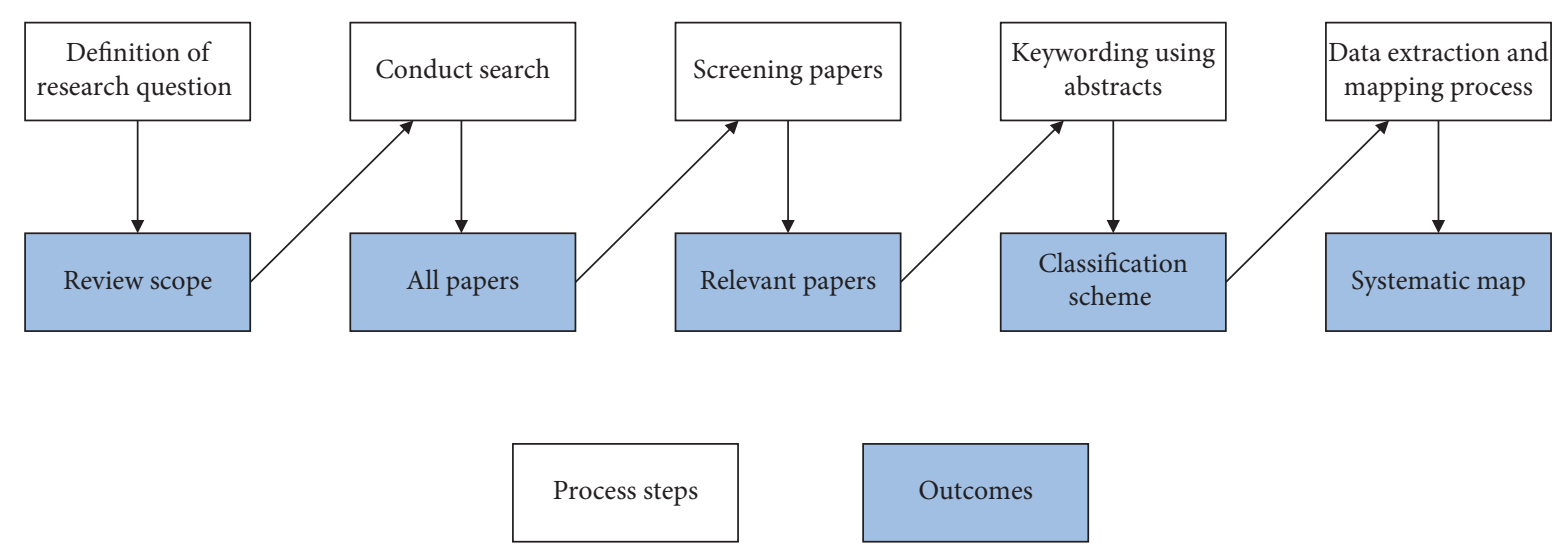

Figure 1: The systematic mapping study.

This question is set to analyze the purpose of the method or tool proposed in the paper.

(vii) RQ7: how are relevant research papers distributed? Basic Principle. We focus on the year the paper was published and the type of publication.

Instruction. The answers to the above 7 questions and data analysis are given in Section 3.

2.2. Collection and Screening of Research Papers. In the process of searching for and selecting research papers, we will encounter some problems.

2.2.1. Search Process. First, we need to select several popular and approximate database ranges in the general database and find the query rules for making searches on the database using its advanced search function. After that, we need to try to determine a query statement and conduct a preliminary search on the database. We need to find a suitable search database and search method, so it is recommended to use the trial search provided by the database. For search engines with different search capabilities for different databases, we need to determine a general query sentence at the level of our research field. For example, in the query, it is expressed as "(plant) and (visualization technology) and (model)"; however, the number of related papers searched by this method is relatively large, and some papers in the search results are not relevant to our needs. Therefore, further screening is required.

2.2.2. Selection Process. Papers are selected in three steps. In the first step, we directly see whether the title can indicate the core idea of the paper. If there is no explanation, we need to read the abstract of the paper. In the second step, after reading the title and abstract, if we did not understand the core idea of the paper, then we need to read the summary or full text. Finally, we need to select articles in the formal screening based on the established selection criteria.

2.2.3. Search and Screening Process. This process has five steps, as shown in Figure 2.

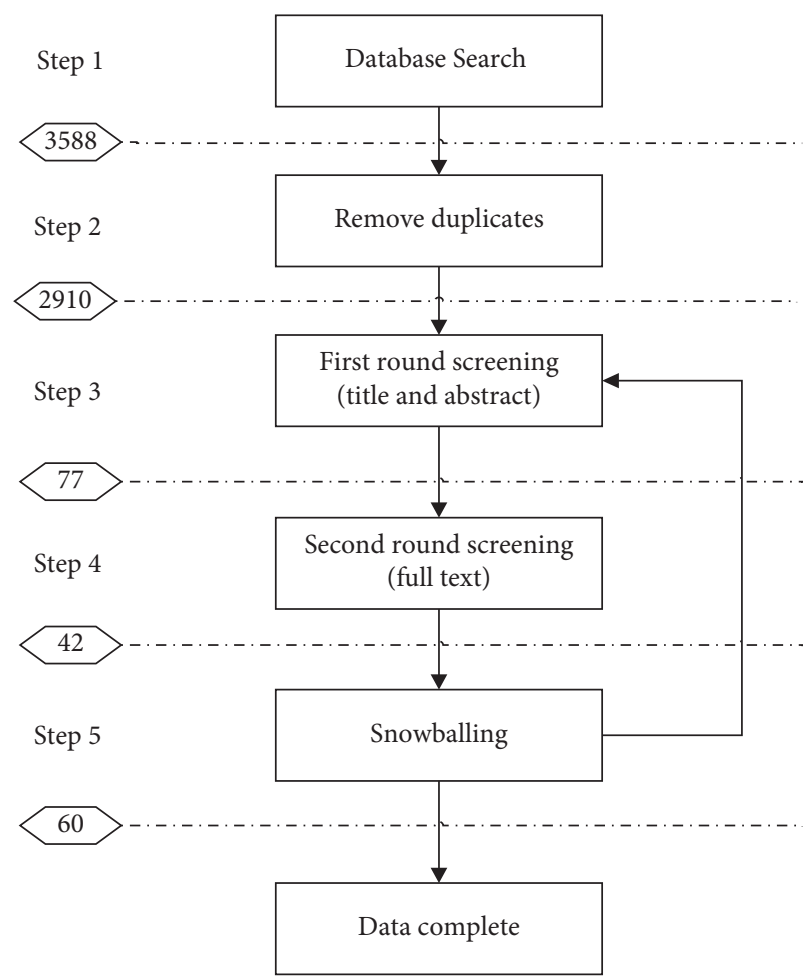

Figure 2: Database search and paper screening process.

(i) Step 1: select a database and search for papers. According to the field of our research subject, we selected four mainstream databases (Table 1) and searched for papers in combination with query sentences.

(ii) Step 2: read the title and abstract of the paper after step 1 to perform the first round of screening.

(iii) Step 3: after completing step 2, to remove duplicate papers, read the title and abstract of the paper for the first round of screening.

(iv) Step 4: if reading the title and abstract still cannot summarize the core meaning of the selected paper, then read the full text. 
TABle 1: Database.

\begin{tabular}{lcc}
\hline Database & Link & Matched scope in database \\
\hline EI & https://www.engineeringvillage.com & Title, abstract, keywords \\
IEEE Explore & https://ieeexplore.ieee.org/Xplore/guesthome.jsp & Title, abstract \\
ACM Digital Library & https://libraries.acm.org & Title, abstract, keywords \\
Web of Science & http://login.webofknowledge.com & Title, abstract, keywords \\
\hline
\end{tabular}

(v) Step 5: the purpose of using the method of snowballing [3] is to supplement the results of the second round of screening. After that, we repeat steps 2-4 for the newly added reference paper.

2.2.4. Paper Search Scope. We use four authoritative databases, ACM, IEEE Xplore, EI, and Web of Science, as the source for collecting papers. Google Scholar is not included in our database search because the use of Google Scholar will overlap with other database search results to a great extent. As far as we know, an excellent paper can only temporarily serve as a milestone in this field; it is not eternal because this research topic is extensive, and new theories and methods will always emerge. According to the reasonable time for system mapping research, we set the research cycle from July 2010 to November 2020, and we searched for papers during this cycle.

2.2.5. Search Query. Kitchenham and Charters proposed the PICO (Population, Intervention, Comparison, and Results) model in order to identify keywords from research questions and formulate search strings [2]. Building a search string in SMS is a very important part. Since the keywords identified from the research question are similar to the keywords identified from the PICO standard, we group them, so we use the PICO model to help us build the search string. In the field of software engineering, population can refer to specific software engineering roles, categories, application areas, and industry groups. Intervention refers to the visualization methodology, tools, techniques, or processes of plant models. Comparison refers to no experience of comparison; certainty; and techniques, tools, and methods for extraction and comparison. Results: there is no need to consider the results of measurement because they do not focus on empirical research to evaluate the mapping of the system. We used the PICO model to determine the sentences describing the research field through each component (Table 2) and extracted keywords according to the guidelines proposed by Peterson et al. [2]. Table 3 shows our extraction process and results. We used "OR" to connect synonyms for a precise search range and "AND" to connect restrictive conditions to form sentences. Our query sentences are expressed as "(theory or modeling) and (plant model or crop model) and (visualization technology or virtual plant) and (application or tool or method).”

2.2.6. Search Guide. Peterson et al. [1] pointed out that it is necessary to make decisions regarding inclusion and exclusion from SMS based on the searched research according to the necessary characteristics, and a complete set of search guidelines needs to be formed for this. Based on the themes of the 3D-PMV field studied in this article, we screened the papers according to the following selection and exclusion criteria:

Selection criteria:

(i) The thesis can provide new theories and methods for the 3D-PMV field or experience and evaluate the methods used in the research field.

Exclusion criteria:

(i) When the title and abstract of this paper cannot provide the core ideas, not only the full text cannot be browsed but also the conclusion cannot be obtained.

(ii) This paper is not in English.

(iii) Search for papers belonging to a book or part of chapter fragments.

(iv) This paper only studies how to build models for plants.

(v) The thesis only focuses on the research of visualization methods, which is completely irrelevant to the research field defined in this article.

(vi) The two-dimensional parametric modeling of the plant model in the paper is not a three-dimensional visualization or an improved method based on three-dimensional modeling.

Analysis. In the search guidelines we established, the selection criteria are based on the research field of this paper in line with the subject of this paper, and the theories, methods, experiences, and evaluations provided by this paper are also for the 3D-PMV field. When searching for papers through the query sentence proposed above, there are a lot of papers in the search results. Therefore, according to the title and abstract of the paper, the core ideas in the 3D-PMV field cannot be provided as the first exclusion criterion. This can not only quickly locate whether the paper meets the selection criteria but also improve paper screening efficiency. In the paper search results, it is necessary to exclude the research keywords mentioned in the book but without experimental theories and methods. Some papers can be preliminarily determined to meet the selection criteria by reading the title and abstract, but after reading the full text, it is found that the paper only studies how to build models for plants or researches around visualization methods that are completely unrelated to the limited research field of this article. Therefore, such papers also need to be excluded.

2.3. Research Classification. After screening some papers, we classify the research according to the abstracts and keywords of the relevant papers. According to the guidelines provided 
TABLe 2: Description of our PICO model combined with 3D-PMV.

\begin{tabular}{|c|c|}
\hline Component & Description \\
\hline Population & Research on the visualization method of 3D plant model. \\
\hline Interventions & Design and development of plant parameter models, research, and application of visualization technology in other fields. \\
\hline Comparison & $\begin{array}{c}\text { We identified different technologies and methods of 3D-PMV, analyzed the purpose of different levels of experimental } \\
\text { results, and compared the research fields of 3D-PMV. }\end{array}$ \\
\hline Outcomes & 3D-PMV can effectively help researchers to study mechanism characteristics through phenotypes. \\
\hline
\end{tabular}

TABLe 3: Extracted keywords from our PICO model.

\begin{tabular}{|c|c|}
\hline Component & Keywords \\
\hline Population & Plant model, visualization method, and virtual plant. \\
\hline Interventions & Design and development of plant parameter models and research and application of visualization technology in other fields. \\
\hline Comparison & Method, analysis, application, plant model, and 3D visualization. \\
\hline Outcomes & Methods, plant models, and visualization. \\
\hline
\end{tabular}

by Peterson et al. [1], we divide the research into seven categories for different research questions. The classification is shown in Figure 3.

2.3.1. Study Type Classification. We classified the selected papers according to the paper classification system proposed by Wieringa et al. [10]. In the set category, each paper does not need to be evaluated in detail by us, so the narrative and classification can be easily determined. Since most of the papers will clarify in the abstract whether the author completed the paper based on a new method or an improvement of a past method, it is easy to determine the classification of this paper. The classification of confirmatory research papers is also easy to determine because we only need to check whether the main components of the paper include stated hypotheses or experimental designs. In this classification system, the research type classification is divided into solution proposal paper, verification research paper, experience paper, evaluation research paper, opinion paper, and philosophy paper:

(i) Solution proposal papers are based on the original solution to improve or develop new technology.

(ii) Validation research papers are a type of papers that do not require a laboratory setting or practice to design experiments, analyze the process, and discuss the results.

(iii) Experience papers belong to the authors' detailed description of personal experience in the process of using related technologies, tools, or methods.

(iv) The evaluation research paper is produced to evaluate the advantages and disadvantages of the research work in practice in the implementation process and the final result.

(v) Opinion papers reflect the authors' personal evaluation opinions on the technology, experiment, tool, or method in a certain research field.

(vi) Philosophical essays discuss new theoretical systems or new methodological proofs for existing things.
2.3.2. Classification of Research Objectives. We summarize the research objectives according to the different papers selected and then classify the research objectives:

(i) The goal of the researchers through the 3DPMV method is to show the growth process of the plant's overall tissue, canopy structure, leaves, and roots.

(ii) The goal of the researchers through the 3D-PMV method is to study plant phenotypic characteristics.

(iii) Some researchers provide new solutions in the field of 3D-PMV and design experiments to verify or use experiments to verify others' methods. Both research objectives are to verify the feasibility of the experiment.

(iv) We call the research goals that cannot be unified into one category other research goals, and the detailed research goals are presented in Table 4.

2.3.3. Model Construction Method Classification. Before researchers study the $3 \mathrm{D}-\mathrm{PMV}$ method, they need to use primitive methods to model plant structures, organs, leaves, or roots. We divide these models into two categories:

(i) Parametric model, which is a plant phenotypic characteristic model following the parameters of plant action mechanism or structural characteristics.

(ii) Geometric model, which is a model built using mathematical function equations, tools, or scanning plant images based on related technologies.

2.3.4. Visualization Dimension Classification. In the field of plant model visualization methods, we need to find the currently popular visualization dimensions. We mainly divide the visualization dimensions into three categories: two-dimensional, three-dimensional, and four-dimensional. Among them, the four-dimensional proposal is based on the original three-dimensional image by adding a time series to generate a computer tomography four-dimensional image. 


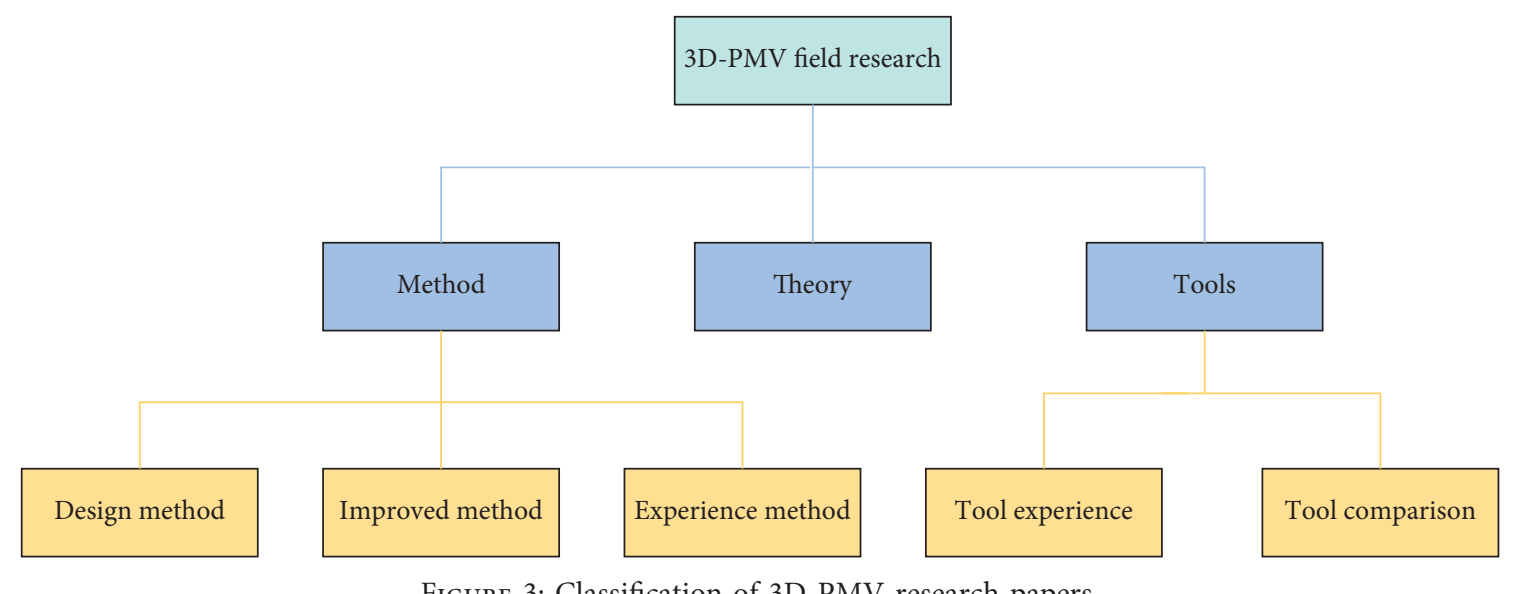

Figure 3: Classification of 3D-PMV research papers.

In order to ensure the popularity of the research problem, we also add it to the visualization dimension category.

\subsubsection{Visualization Purpose Classification. We categorize} the visualization purposes according to the nature of the $3 \mathrm{D}$ PMV functions developed by different researchers. Generally, visualization is divided into dynamic and static categories. The following describes the detailed distinction between these two categories.

\section{Static visualization:}

(i) Visualization demonstration type: in order to pursue a realistic visualization effect, when performing virtual simulation modeling of plant morphology, the emphasis is on using morphology visualization technology or simulating real plant morphology modeling. The purpose is to study how to visualize plant characteristics, such as canopy structure, leaves, and roots.

(ii) Morphological description type: when exploring the morphological and structural changes that occur in the process of plant growth, it is usually possible to construct a geometric and parametric static virtual plant model that reflects the changes in plant morphology on the basis of experimental observations. According to the morphological characteristic parameters of this type of model at different growth stages, the visual demonstration of plant shape changes is realized in order to show the static growth and development process of each stage of the plant more intuitively and digitally.

(iii) Visualization-morphological combination: we combine the corresponding functions of the above two types of models to produce static visualized plant combination model classification. The models belonging to this classification can not only meet the needs of visualized plant morphology display but also provide analyses and references for the physical changes at different stages of plant development and growth through the visualized results.
Dynamic visualization:

(i) Phenotypic structure change type: we classify plant visualization models that simulate the process of plant morphological structure change in a dynamic manner, aiming to provide an intuitive scientific basis for dynamically grasping the topological structure of plants and other organs and tissue morphological changes.

(ii) Mechanism and function change type: since the growth and development process of plants is the result of the interaction between external environmental factors and genetic genes, we construct a visual plant model classification that reflects the growth mechanism of plants. This type of model explores the action rules of plant external environmental factors and internal mechanisms through dynamic visual simulation.

(iii) Structure-mechanism interaction type: not only the external morphological structure of plants changes with the growth stage but also the internal mechanism of plants is constantly changing. Therefore, the research on the visual model of plant phenotypic structure and internal mechanism is a new category. This type of visualization research is conducted to show the mechanism changes in different growth stages of plants.

2.3.6. Classification of Method Papers and Tool Papers. If papers propose a new experimental design, technical method, or improvement of a method, we classify them as a method paper. For papers that pay attention to describing the process of using software and tools to achieve 3D-PVM effects, we classify them as tool papers.

2.3.7. Distribution Classification. We follow a general classification scheme to classify publication information, such as the publisher, publication year, and author of the paper. The paper site classification is divided into international journals and international conferences. 


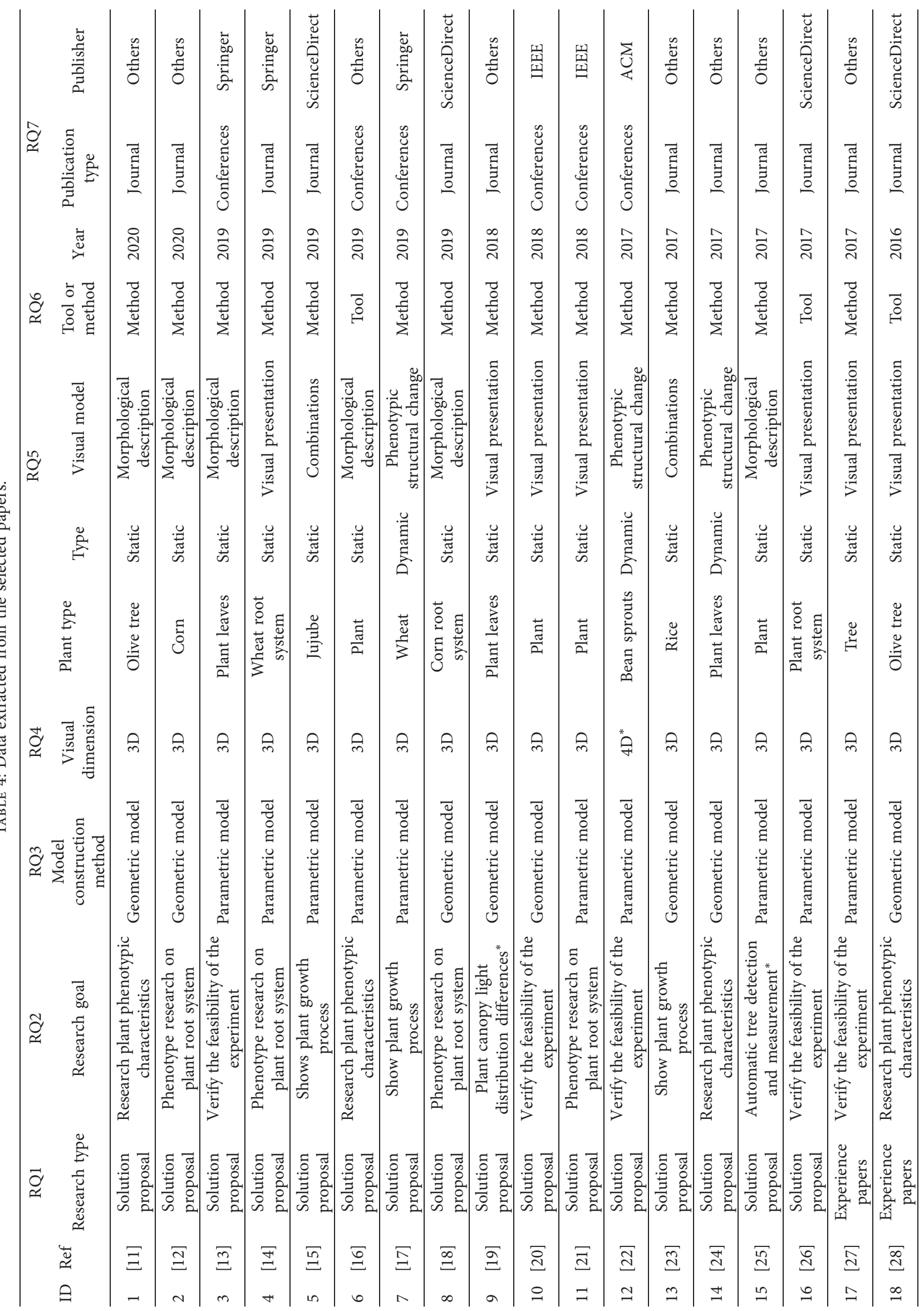




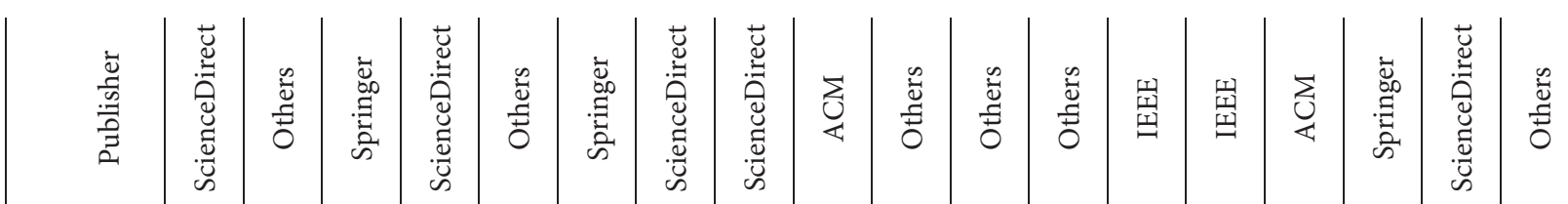

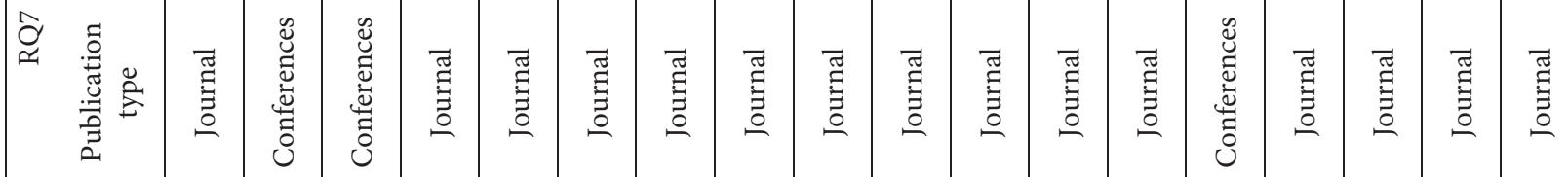
荧

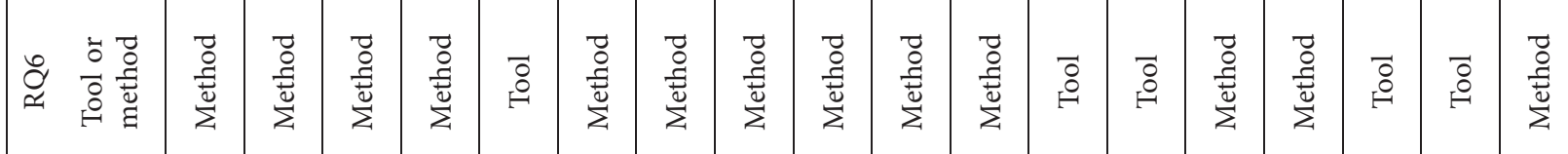

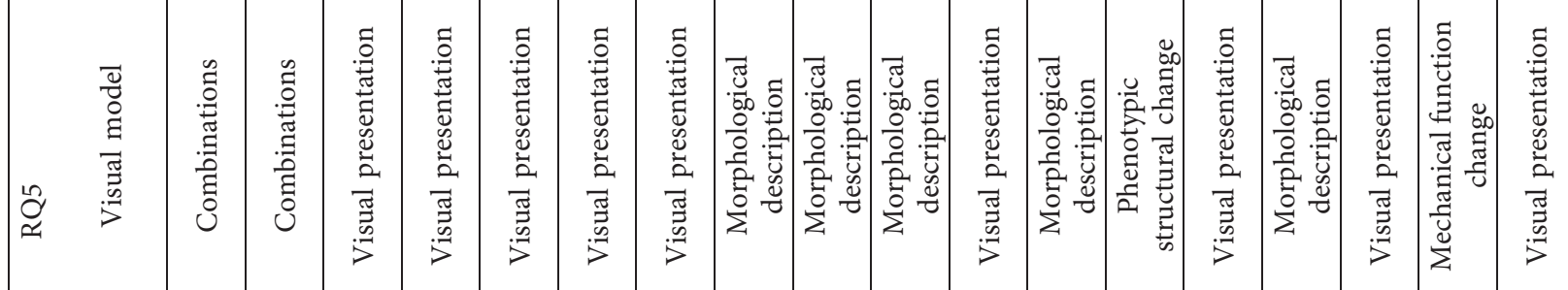
茎 苞

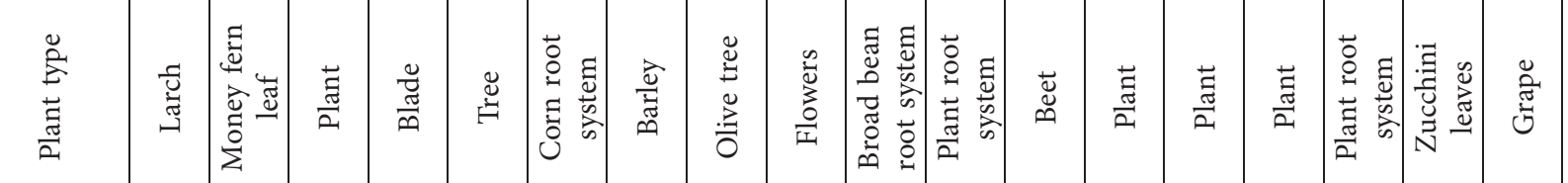

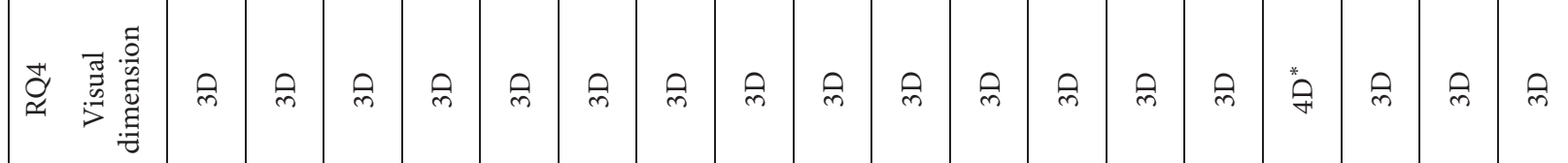

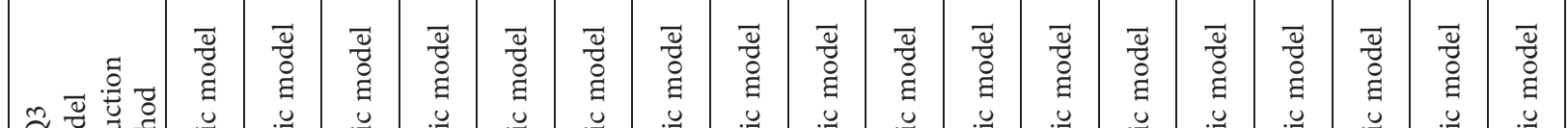

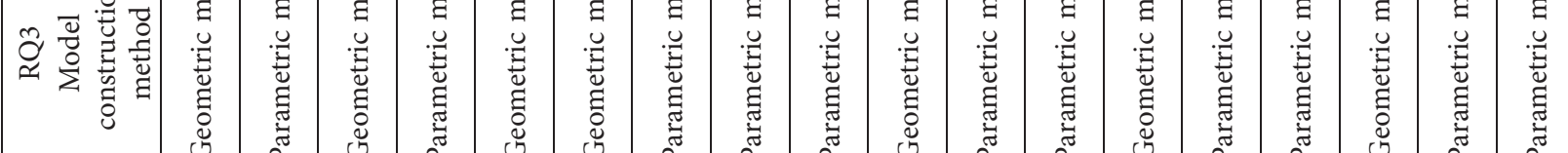

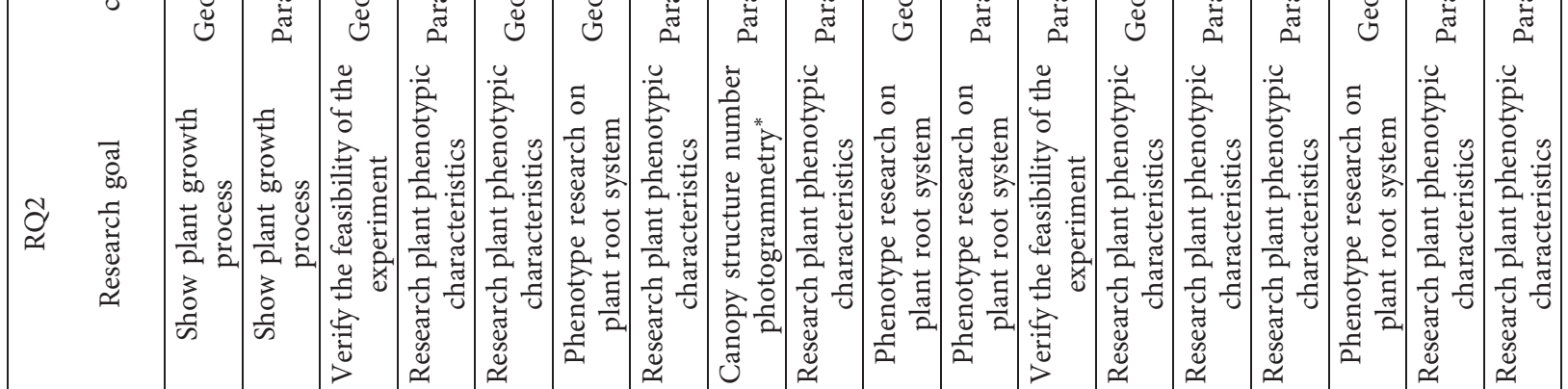

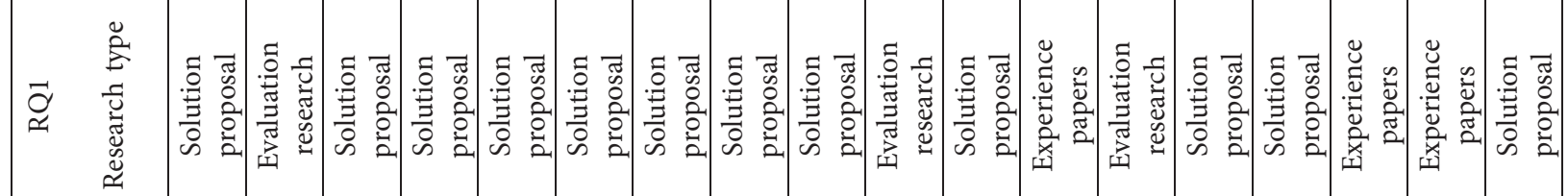

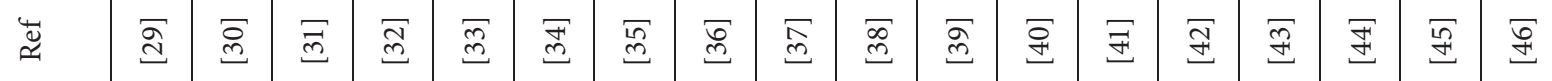

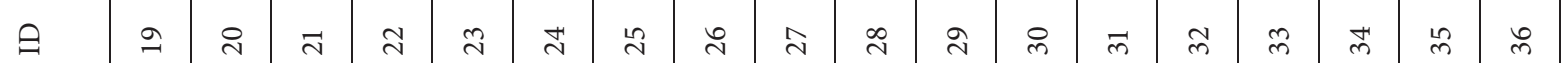




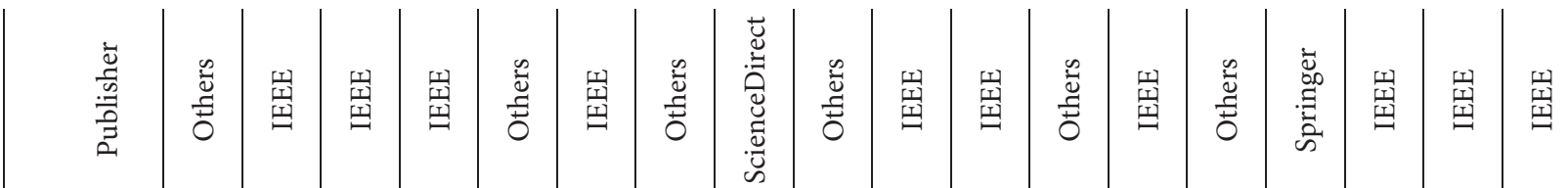

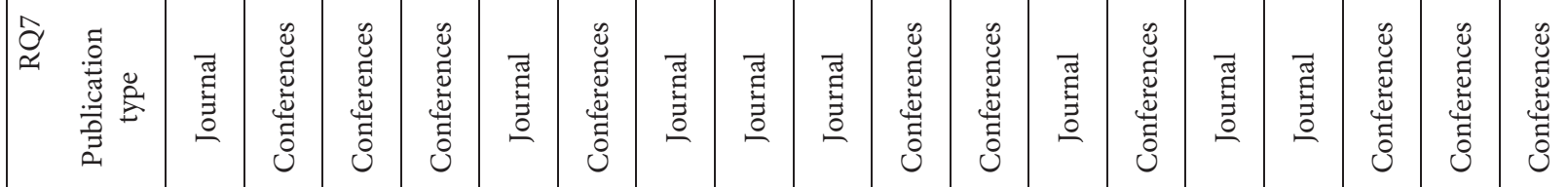

䒕

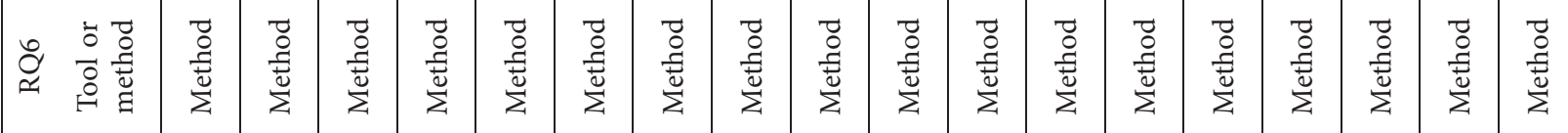

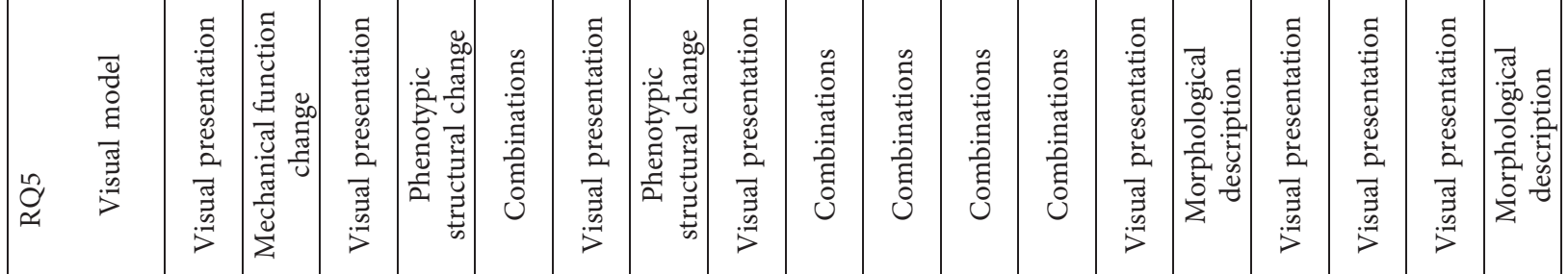

茎

苛

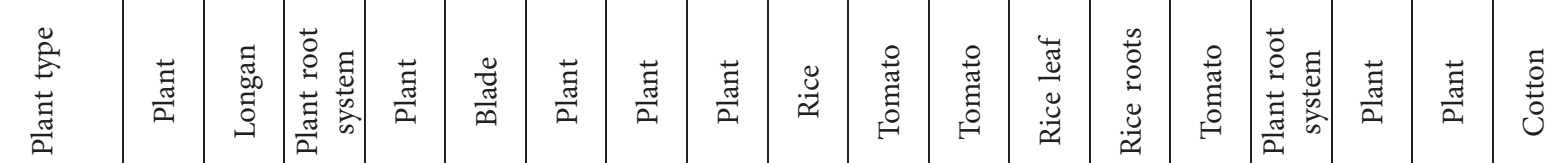

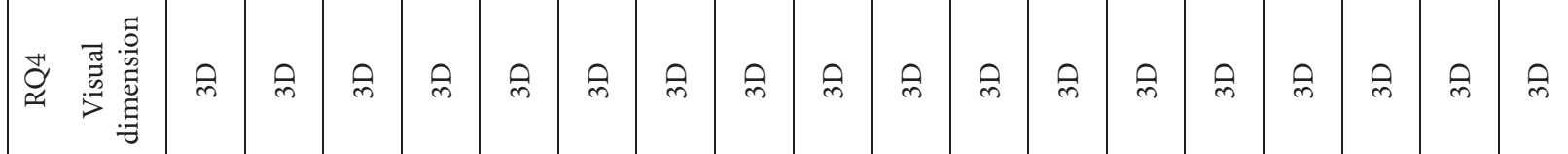

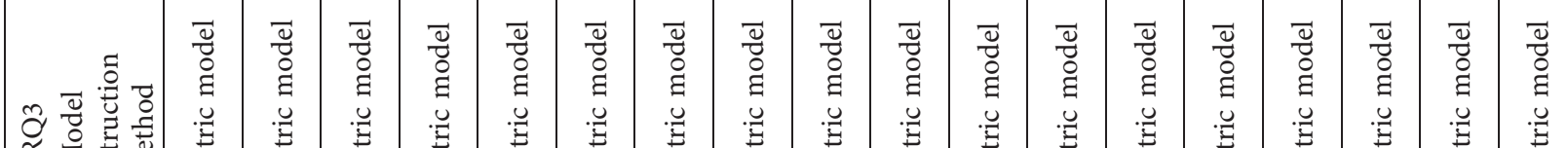

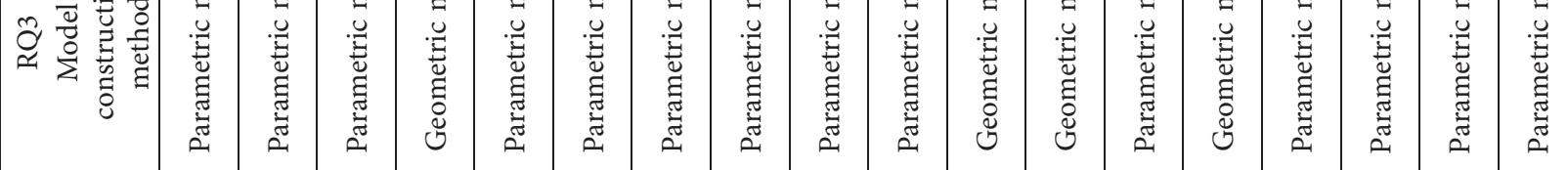

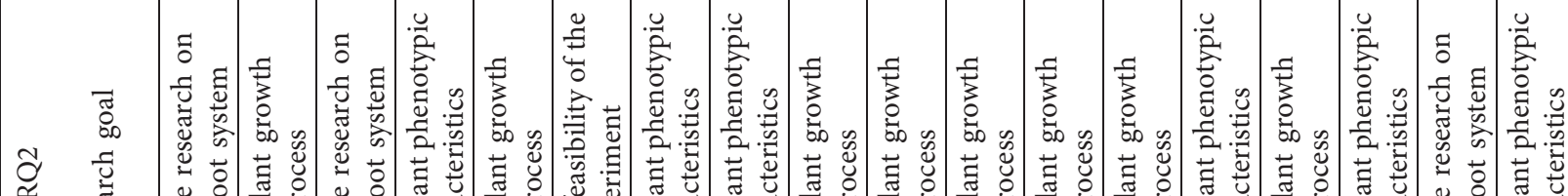

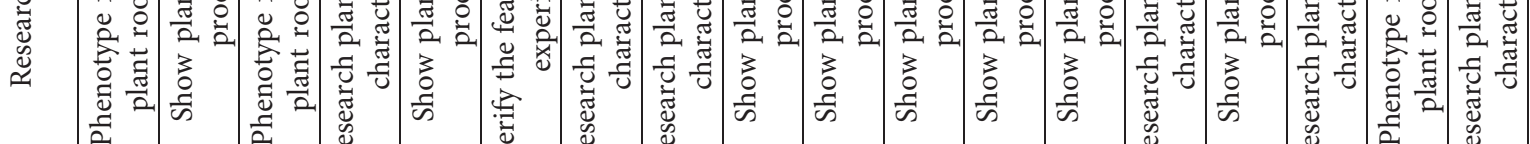

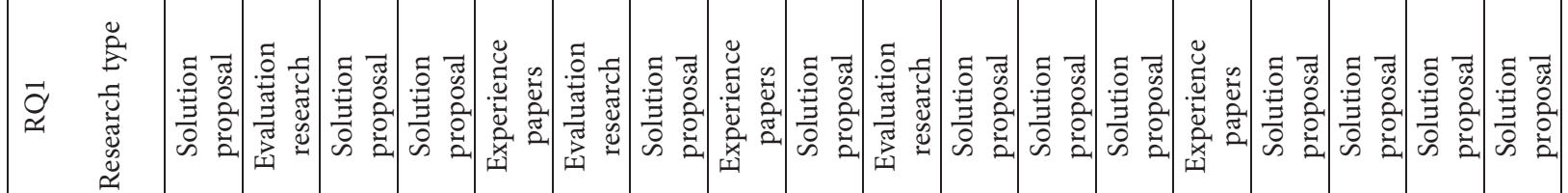

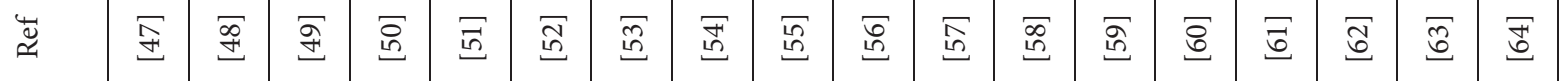

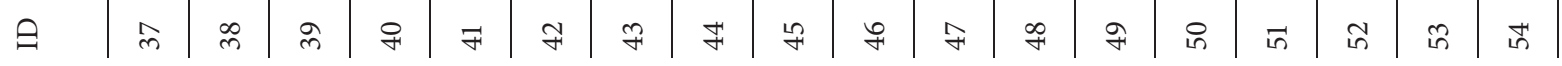




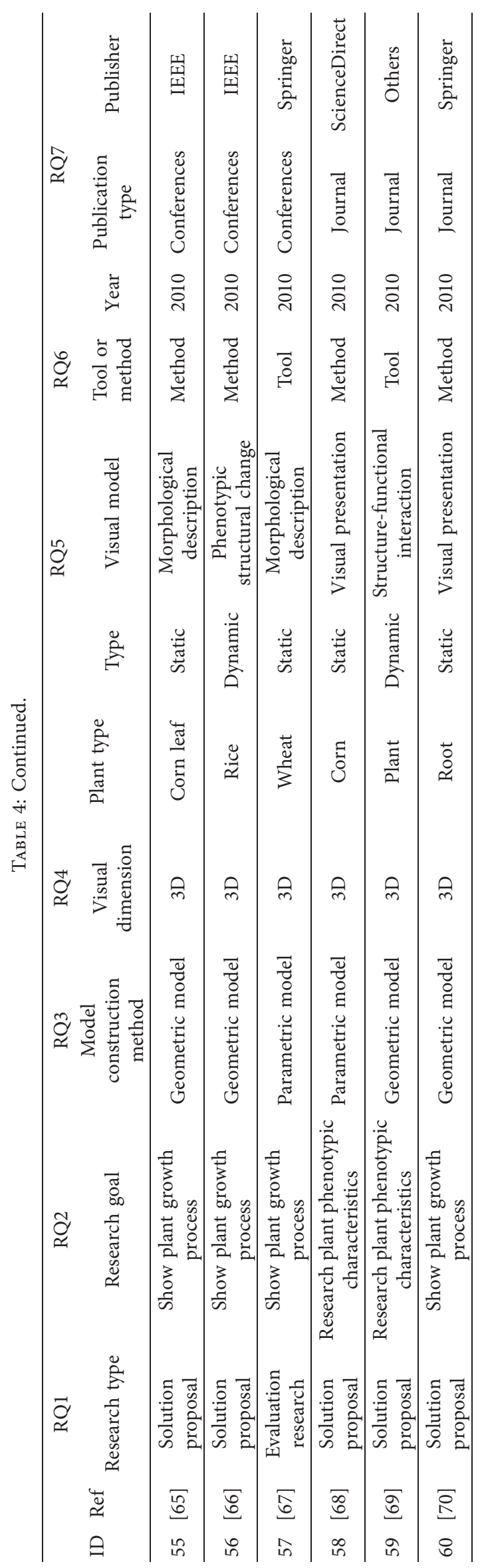


2.4. Data Extraction and Research Mapping. After reading each of the selected research papers, we fill out the electronic form according to the research questions raised. The fields of the form are used to record the data of the selected research and then extract the data to answer the research questions. The table fields include the type of research to answer RQ1; answer the research objectives of RQ2; answer RQ3's modeling method; answer the visualization dimension of RQ4; answer the visualization purpose of RQ5; and answer the details of the methods and tools of RQ6. The data extraction results are shown in Table 4. Finally, the extracted data are mapped to the classification problem, combined with the visual analysis of the form of graphics.

\section{Result}

3.1. Search Options and Snowball Results. The database search process is shown in Figure 2. We retrieved 3588 papers from four databases. We removed the overlapping parts of the database search results and retained 2910 papers. In the first round of screening, we retained 77 papers. After the second round of screening, we retained 42 papers and identified 18 papers through reference tracking [3]. Finally, the number of selected papers was determined to be 60 .

3.2. RQ1: What Types of Research Have Been Conducted in This Research Field over a Period of Time and How Has It Developed? According to the research types selected from 2010 to 2020 based on the process in Figure 2, we found that solution proposals accounted for the largest proportion of the research types, accounting for $75 \%$ of the total 60 papers, followed by experience papers, accounting for $13 \%$ of the total papers. Evaluative research papers accounted for $12 \%$ of the total. There are no validating research papers, opinion papers, or philosophical papers in our paper type classification.

As shown in Figure 4, the number of papers of the solution proposal type has increased since 2010, and the number of papers stabilized after 2012. These types of research papers account for $75 \%$ of all types, which means that most researchers in the field of 3D-PMV focus on the improvement of the original method or the proposal of new methods. At the same time, as the publication of solution proposal type papers tends to stabilize, the corresponding evaluation research papers and experience papers are almost the same. In Figure 5, it can also be observed that the proportions of these two types of papers differ by only $1 \%$. It is not difficult to see that the experience, application, and evaluation of improving the original method or proposing a new method in this field are of considerable importance.

During our search and screening of papers, no verification research papers, opinion papers, or philosophical papers were found. In the future, researchers in the field of 3D-PMV need to explore more innovative methods to realize 3D plant visualization and put forward more concepts and perspectives in this field.

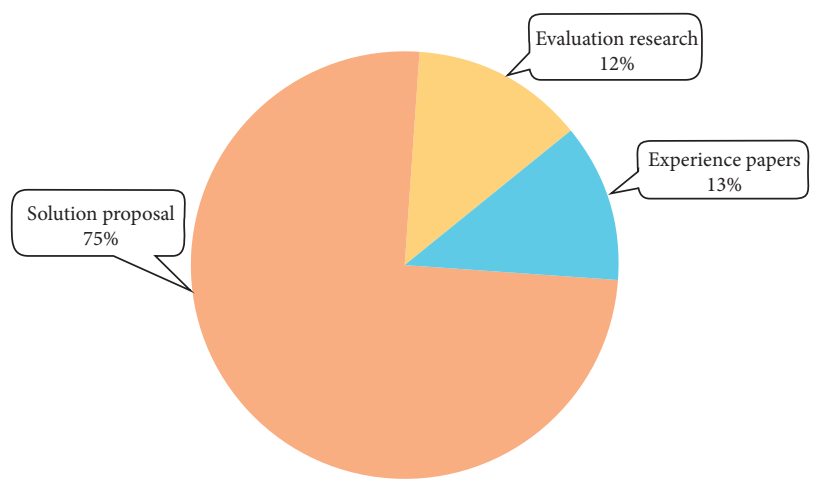

FIGURE 4: Research type classification.

3.3. RQ2: What Are the Research Goals? Based on the results of the papers we selected, the summary research regards five main research goals. The research of plant phenotypic characteristics is mainly aimed at extracting and modeling the phenotypic structure characteristics of plants, and the research shows a detailed and complete phenotypic structure of the whole or part of the plant. The presentation of the plant growth process is the display of different morphological changes of plants according to different growth stages of plants, which is helpful to the exploration of the mechanism changes of various growth stages of plants. Some research on the visualization of plant roots was found in the selected papers, so the research targets were classified as phenotype research on the plant root system. This research goal focuses on the study of visualizing the growth of plant roots in the soil and the improvement of root segmentation methods. The research goal of the verification of the feasibility of the experiment regards the experimental verification of new methods or improved methods proposed by researchers in related fields, focusing on the feasibility of the method to evaluate and promote the research method. Those that cannot be unified into a type of research objective are classified according to other categories. The research objectives of this classification are detailed in Table 4 and marked with the symbol “*”.

As shown in Figure 6, the research target is the type of research of plant phenotypic characteristics, which accounts for $35 \%$ of the overall research target. Aiming at the simulation of plant phenotypic characteristics, researchers complete various studies on plant phenotypes according to different methods or tools (see, e.g., $[16,24,28,35,45,46])$. The research target showing the growth process of plants accounted for $28 \%$. The related research of this target can provide changes in the internal mechanism of plants and achieve dynamic prediction effects on plant growth conditions (see, e.g., [17, 29, 48, 55, 61]). Since the morphological structure of plants at different growth stages is quite different and the structure gradually becomes more complex, this type of research is accompanied by huge challenges. In our screening, the research on $3 \mathrm{D}$ visualization of the plant root system accounted for $18 \%$, which is also a non-negligible part. For example, the difference in soil environment affects plant growth as reflected by the root system (see, e.g., [14, 49]), as observed in research on improved methods used for plant root system segmentation and root 


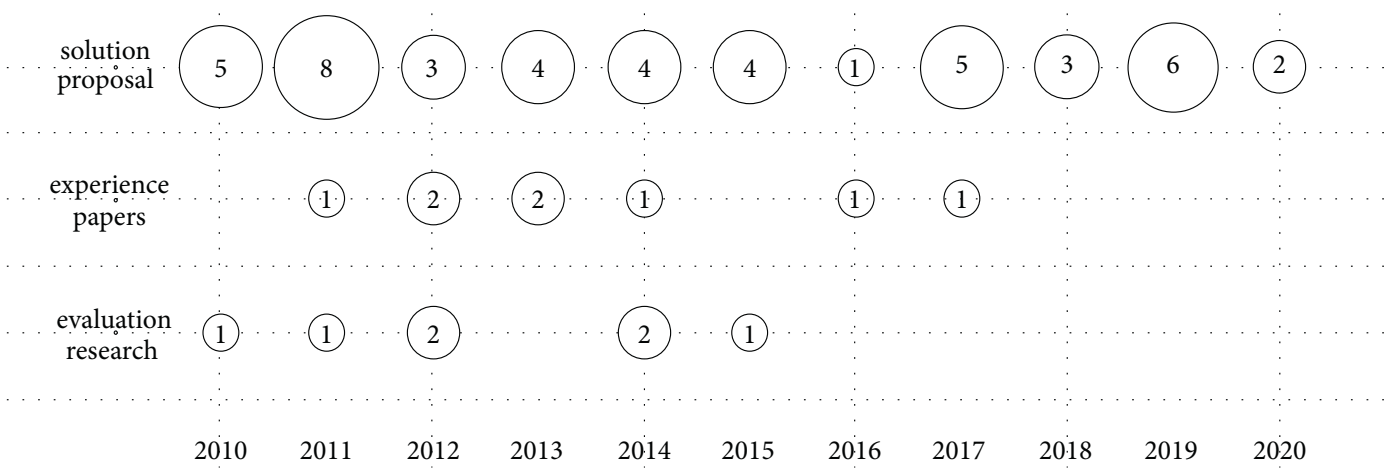

FIgURE 5: The number of research types per year.

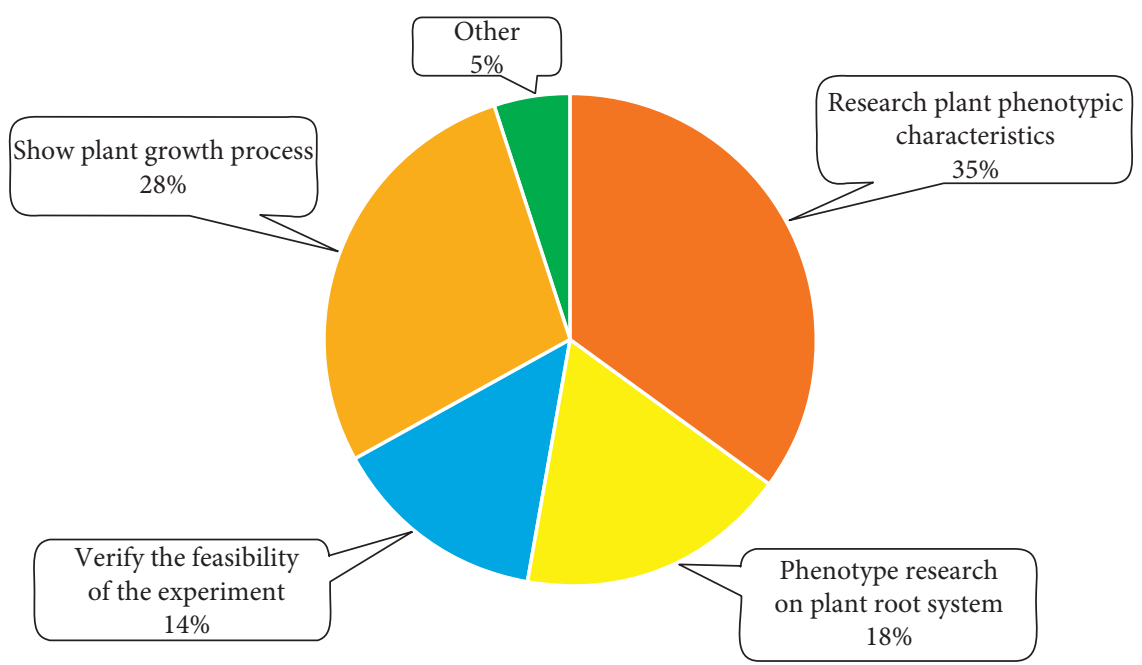

Figure 6: Research goal classification.

system extraction in non-destructive soil environment visualization (see, e.g., $[21,34,39,44])$. The research target to verify the feasibility of the experiment accounts for $14 \%$ of the total, and the experimental verification is mainly designed for the proposed improvement method or to provide an evaluation in order to compare the original method and the existing method (see, e.g., $[13,20,22,26]$ ). We classify the research goals of determining plant canopy light distribution differences, automatic tree detection and measurement, and canopy structure number photogrammetry into other categories (see, e.g., $[19,25,36])$, occupying $5 \%$.

\subsection{RQ3: Which Studies in 3D-PMV Use Traditional Model} Building Methods? In this work, it was observed that researchers will first establish the parameter model or geometric model of the plant in an original way before finally realizing the visualization results of the model. Parametric models account for $62 \%$ of the total (37 out of 60 ). The establishment of the model relies on the extraction of plant morphological parameters, and the model of the plant phenotype or growth stage is established based on these parameters. The proportion of the geometric models is 38\% (23 out of 60 ). A virtual model is established according to the research direction after mathematical equations are established by analyzing the geometric forms of plant phenotypic organs. The establishment of these two types of models is carried out to prepare for the subsequent visualization of plant models.

In the study of plant phenotype modeling, the establishment of parameter models is relatively common. For example, Behmann et al. [32] used a hyperspectral camera to scan plants in order to extract phenotypic parameters, and Paulus et al. [35] used laser scanning technology. Plant morphological parameters are extracted to capture the $3 \mathrm{D}$ plant structure. The geometric models of plant morphology, organs, and root systems rely on the establishment of mathematical equations. For example, Li et al. [23] used the number of pixel columns and rows of the leaf image to convert the real-coordinate quadratic function equation of each pixel to establish the leaf geometry model. Han et al. [29] used the measured crown profile data of each tree to compile a visualization model to form a tree visualization geometry.

3.5. RQ4: Which Dimension Realizes the Visualization of the Plant Model? According to the papers we selected covering a period of 10 years, it was found that $97 \%$ (58 out of 60 ) of the papers realized the $3 \mathrm{D}$ visualization of plant models, and 
only 3\% (2 out of 60 ) of the paper researchers named it according to the $4 \mathrm{D}$ visualization. Researchers added a time series to the original three-dimensional images to generate four-dimensional computed tomography images, so 4D CT technology was proposed [22, 43]. According to our screening results, no research regarding $2 \mathrm{D}$ plant model visualization was found.

3.6. RQ5: What Are the Categories of 3D Plant Visualization Models? Due to the different nature of the 3D-PMV functions developed by different researchers, we classify the visualization purposes. We have stated in Section 2 that its types are generally divided into two categories, dynamic and static. These two types are divided into six categories in detail. As shown in Figure 7, the first (41\%) types are visual presentations; the second (27\%) are morphological descriptions; the third (15\%) are combinations; the fourth $(12 \%)$ are phenotypic structure changes; and the last type accounts for 3\%. The mechanism and function of the variability type account for only $2 \%$ of structure-function interaction. The type of visualization presentation focuses on using methods such as morphological visualization technology or realistic modeling technology to explore how to express characteristic information such as the overall structure of plants and the morphology and texture of organs. For example, Chen et al. [14] used the proposed method of root system architecture (RSA) digitization and modeling to demonstrate the skeleton structure of wheat roots, and Wen et al. [19] used hierarchical parametric veins and margin (HPVM) to construct a parametric surface leaf model. We classify the visual description of the morphological feature model of plants, organs, or roots at a certain stage of the plant growth process into static/morphological description. For the research field of visualizing crop root morphology, the ability to extract large and complex root morphological features plays an important role in plant breeding research [18]. The combination type not only meets the needs of visual demonstration of plants but also needs to provide visual analysis and reference for the morphological changes of plants at different growth stages. The digital information of the corresponding growth stage of the plant is extracted through data collection and image capture to study the phenotype and structure of the plant [23]. This category occupies a small proportion in the functional classification of visualization models. We analyzed the reasons for this result: many related studies focus on the continuous improvement of the visualization method of a certain organ and tissue of the plant, but only $28 \%$ of the studies focus on the modeling of plant growth process; in this $28 \%$ of the research, it is necessary to remove part of it to improve the accuracy of the growth stage model. The difference between the phenotypic structure change type and the morphological description type is obvious. The former is used to visualize the morphological changes in each growth process of the plant, while the latter is only used to visualize the morphology of a certain growth stage. For example, in the experiment to study on the influence of plant canopy structure on canopy bidirectional reflectance and bidirectional reflectance distribution function, the development of different plant parts during plant growth is considered [41]. According to our selection, the functional change type accounts for $3 \%$, while the structure-function interaction type accounts for only $2 \%$. In future research, researchers should provide more references for plant mechanisms combined with dynamic visualization. The dynamic type of model visualization also accounts for only $17 \%$ of the total, indicating that the related research on visualization in this field tends to be the static type.

\subsection{RQ6: How Many Tool Papers and Method Papers Were} Screened Out? Among papers written between 2010 and 2020 , we screened out 50 method papers accounting for $83 \%$ of the total, and 10 tool papers accounting for $17 \%$ of the total. Among the 50 method papers that we selected, the main purposes of these methods are as follows: (1) to improve the original method to make the model visualization more realistic and (2) to propose a new method to provide more references for the current research field. For example, for the first time, a 3D geometric model of corn stubble was established in situ by X-ray computed tomography (CT) [12], and a new method for quantifying the spatial distribution of mature plant roots in the field using a 3D digitizer was developed [34]. Breaking the conventional static plant model, based on research and observation, a dynamic law model reflecting the structure of crops has been established [65]. After sorting out all of the tool papers, we found that the tools used by these researchers are different, for example, using 3D point cloud data measured from real plant organs to create a 3D template of plant organs [16]. The research provides an adaptable numerical aid to simulate the interaction between plant roots and soil and to predict the time of cutting stubble in order to determine the resistance of the tool [26]. The leaf wilt index is defined from the spectrum of the 2D Fourier transform through mathematical tools, where the leaf is equivalent to the mathematical surface in the $3 \mathrm{D}$ space [45].

\subsection{RQ7: How Are Relevant Research Papers Distributed?}

3.8.1. Distribution by Publication Type. We set the paper publication type as international journals and international conferences. In total, $63 \%$ (38 out of 60 ) of the authors chose journal publications, and $37 \%$ (22 out of 60 ) of the authors chose conference publications. As shown in Figure 8, we extracted both the publication type and the publisher label and found that the number of journal papers published by IEEE publishers was considerably lower than that of conference papers, and the number of journal papers of other popular publishers was higher than the number of conference papers. More authors chose journal publications over conference publications, indicating that there are fewer conference platforms in this field. After reading these papers, it can be easily observed that the methods and techniques in this field are updated slowly. Similarly, some methods and techniques can be updated less iteratively. 


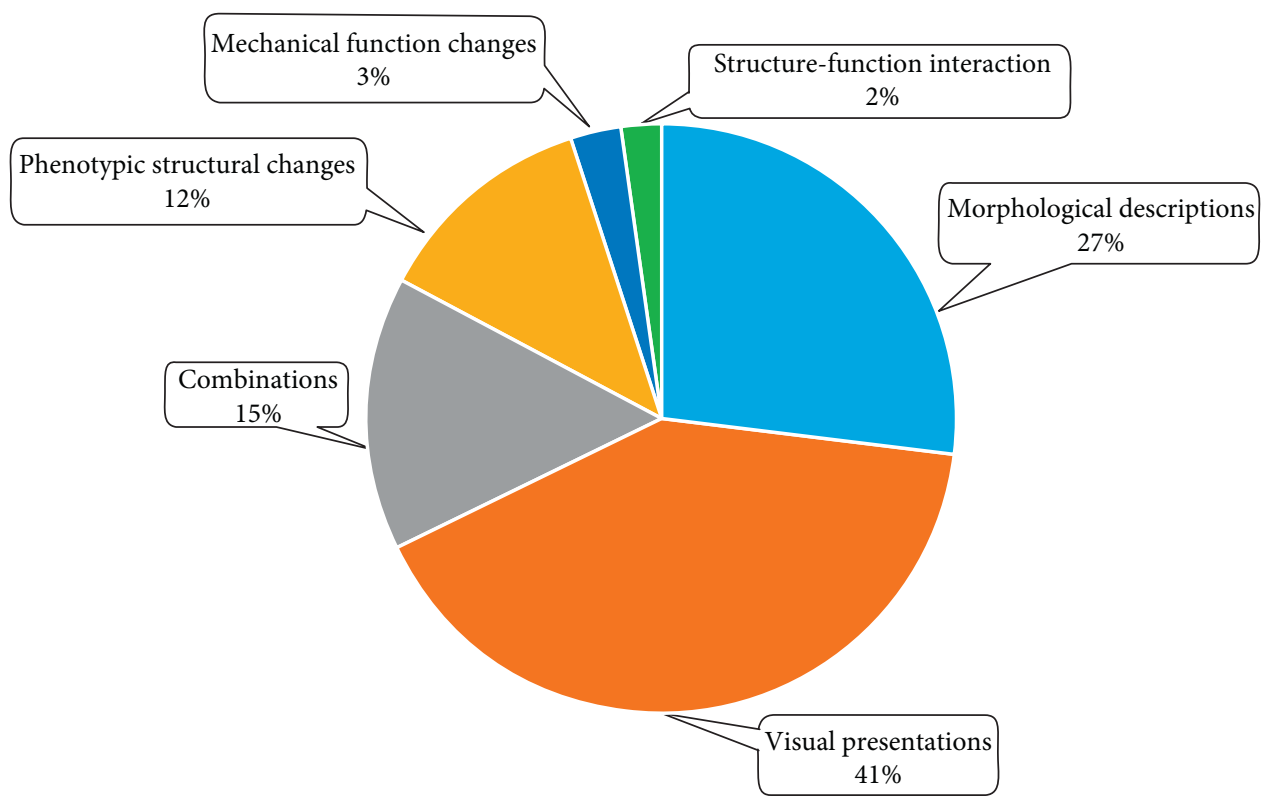

FIGURE 7: Plant visualization model classification.

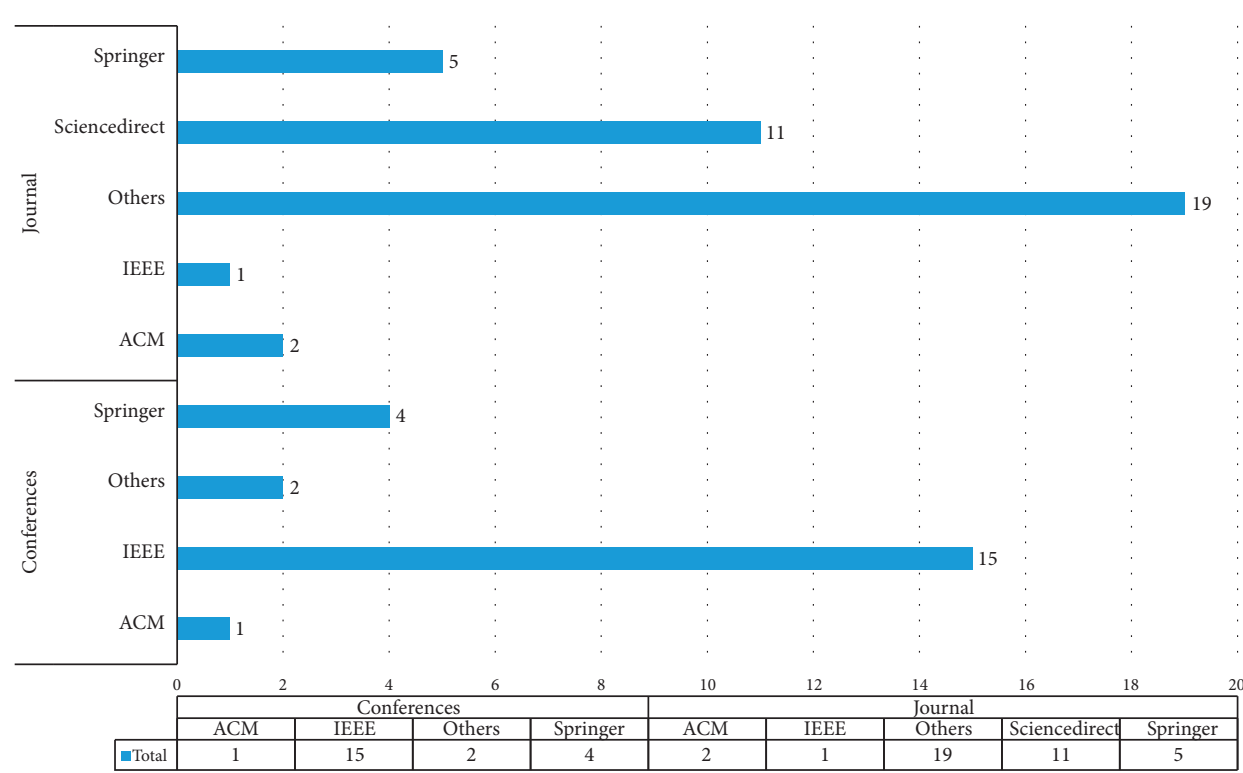

FIgURE 8: Statistics of publishers by type of published papers.

3.8.2. Distribution by Publisher's Category. We counted the papers of different publishers in the 3D-PMV field, as shown in Figure 9. RQ5 type, RQ5 visualization model classification, and data capture analysis provide the distribution of the visualization model categories published by publishers in this research field.

We found that IEEE published 16 articles of different visualization types, including static categories: morphological description (2), visual presentation (8), and combination (2); dynamic categories: phenotypic structure changes (3) and mechanism functions change (1). ScienceDirect published 11 articles, including static categories: morphological description (2), visual presentation (6), and combination (2); dynamic categories: mechanism function change (1). ACM published three papers: static morphological description (2) and dynamic phenotypic structure change (1). Springer published 9 papers: static visual presentation (6), morphological description (2), and dynamic phenotypic structure changes (1). Other publishers published 21 papers, including static visual presentation (6), morphological description (7), combined type (5), dynamic phenotypic structure change (2), and structurefunction interaction (1). Papers published by the wellknown IEEE, ScienceDirect, ACM, and Springer publishers account for $65 \%$ of the total, indicating that papers in this research field can be recognized by popular publishers, which will help the future development of this research field. 


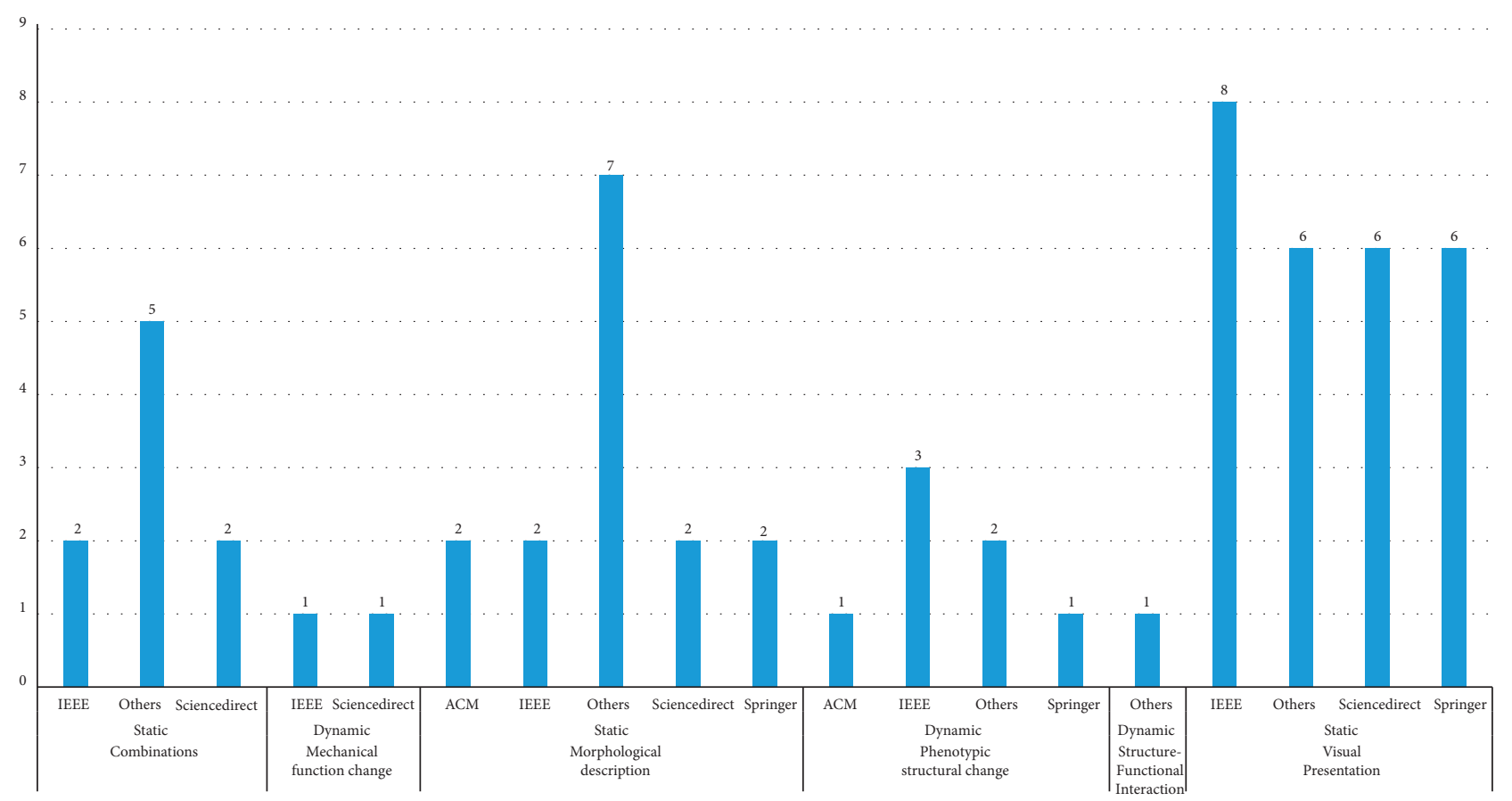

FIgURe 9: Classification by number of papers published by publishers and visualization type.

3.8.3. Distribution by Year of Publication. As shown in Figure 10, from 2010 to 2016, the number of publications each year showed an increasing trend first and then a decreasing one. In the years that we counted, we found that 2011 had the largest number of publications. In the five years after 2011, the number of publications decreased year by year and then increased in 2017. However, the number of publications fell again in 2018. The change in the number of publications in the period of from 2019 to 2020 is the same as that in the previous two years. Only papers before November 2020 are selected for this work. According to the query sentence "(theory or modeling) and (plant model or crop model) and (visualization technology or virtual plant) and (application or tool or method)," search the ACM, IEEE Xplore, and EI database for the number of papers published in the first half of 2021, and 264 papers from ACM, 9 papers from IEEE Xplore, and 27 papers from EI are retrieved. Therefore, according to the recent $3 \mathrm{D}$ virtual plant research trends, we believe that the number of publications in 2021 will exceed that in 2020, so we believe that this field has great potential.

\section{Discussion and Challenges}

In this section, we discuss the research system in the field of 3D-PMV and clarify the challenges encountered in this field.

4.1. 3D-PMV Field Research System Discussion. The current research on plant $3 \mathrm{D}$ visualization technology mainly focuses on three aspects of the plant mathematical model, computer tomography, and plant image feature extraction. In the research on the visualization method of three-dimensional plant organ structure 10 years ago, many related works needed to establish correct parameters or geometric mathematical models of organ tissue structure in different growth stages. In this model, the vectorization of plant organ morphology and growth process is only achieved through a few explicit biological parameters. Using computer graphics technology, combined with $\mathrm{VC}++$ and OpenGL to provide an integrated development environment, the simulation and visualization of the growth process of a plant or organ structure are realized on the computer $[32,66]$. Some use digital photography and commercial photogrammetry tools to extract the parameters of the image of a certain growth stage of the tracked plant and establish a visualization model [68]. The former is to statically visualize the specific period of the growth stage of the plant organ structure, and then a dynamic visualization model for the plant organ structure is proposed [43]. In contrast, the latter model method is more interactive and flexible. However, most X-ray computed tomography processing protocols are only used to detect simple and small roots. Therefore, in recent studies, a new method for extracting the ability of large mature corn roots from undisturbed soil monoliths of X-ray $\mu \mathrm{CT}$ volume has been proposed [54]. This method can distinguish roots to a diameter of $200 \mu \mathrm{m}$, opening up new possibilities for the study of large and complex root systems, and it may become a valuable new tool in plant breeding research. Later researchers added a time series to the original three-dimensional image to generate a four-dimensional computed tomography image. Therefore, a 4D CT technique was proposed to detect plants in a CT scan within a few days at a fixed time interval, allowing for a four-dimensional volume image to be obtained [12]. Instead of modeling the entire plant root system, using an optimized plant root system segmentation method, the smallest and inaccurately initialized image slices in various directions from the smallest 


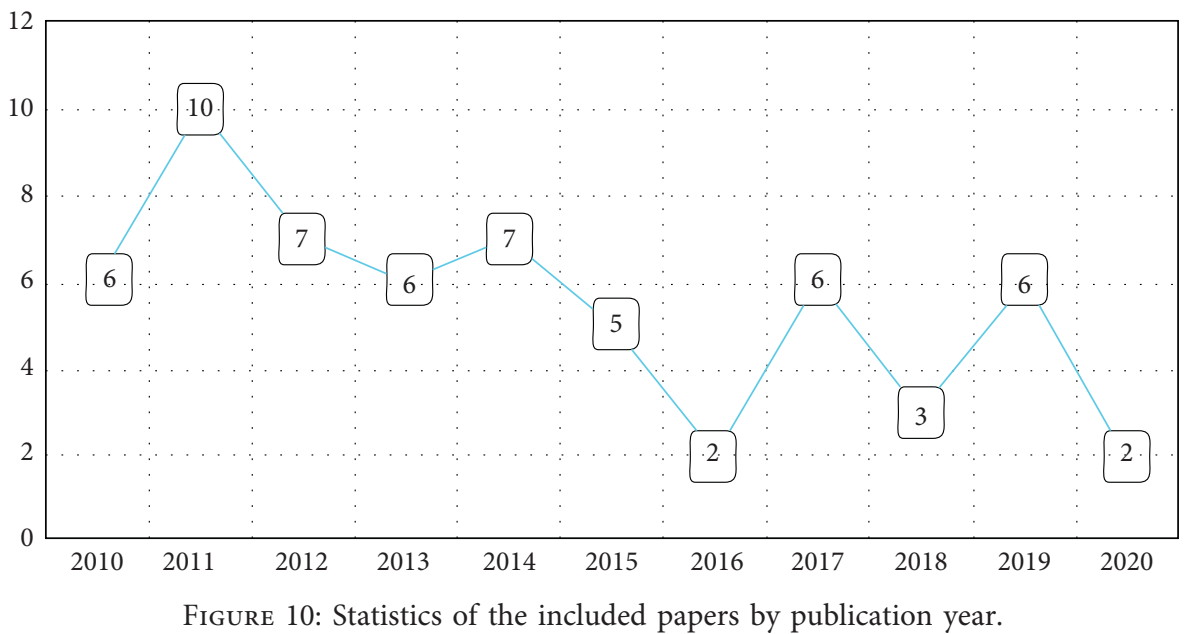

X-ray CT volume were demonstrated [21]. This method considers the distribution of the root area and non-root area of the same plant in the complex soil environment. Moreover, 10 years ago, in the field of plant image feature extraction, researchers used portable high-resolution scanning lidar to generate accurate $3 \mathrm{D}$ images of tomato canopy [40]. Due to the constraint that the laser beam of the lidar equipment cannot illuminate and scan all of the leaves, this method cannot handle the inevitable occlusion between tomato leaves, and the overall $3 \mathrm{D}$ visualization image effect is not ideal. A concept study confirmed that the hyperspectral image captured by the hyperspectral imaging device was used to construct a $3 \mathrm{D}$ plant model in a laboratory environment [41], but this method could not yet generate an overall three-dimensional plant model. In the field of modeling and visualization methods for studying part of the canopy and overall phenotype structure of trees, the use of drones to take high-resolution images to build digital surface models is often used, and then image analysis and processing techniques are used to generate individual tree and entire area tree visualization images $[39,50]$. Using image feature extraction technology, it is also possible to extract static appearance attributes of plants from images containing lesion shapes, diffuse reflectance, and texture features [24]; visually simulate and predict the process of plant disease; and provide decision making in the field of planting.

4.2. Challenges in the Field of $3 D-P M V$. According to the selected research types, it was found that solution proposals account for the largest proportion of the research types, accounting for $75 \%$ of the total 60 papers, followed by experience papers at $13 \%$ and finally evaluative research papers at $12 \%$. The above data show that in the field of $3 \mathrm{D}-\mathrm{PMV}$, researchers pay attention to the improvement of original methods or the proposal of new methods, but they still need to provide more experience and evaluation of existing methods. No verification research papers, opinion papers, or philosophical papers were found in our classification of papers. Therefore, researchers in the field of 3D-PMV need to explore more innovative methods and put forward more concepts and perspectives in this field.

From the results of analyzing the types of visualization models, it was found that most research focuses on statically visualizing plant phenotypic characteristics, and the research on $3 \mathrm{D}$ visualization of plant growth stage models accounts for a small number of studies. In particular, the mechanism function change type accounted for $3 \%$, and the structurefunction interaction type accounted for $2 \%$. Therefore, the 3D-PVM field may have limited research on the realization of plant dynamic visualization methods in the future, which may cause researchers difficulties. It is necessary to provide more visualization methods of studying the combination of plant-intrinsic mechanism and phenotypic characteristics. In the future, we believe that more studies can be conducted to solve this problem, which will help in understanding the mechanism of the various growth stages of plants at a deeper level.

\section{Conclusion}

The methods in the field of 3D-PMV have been widely used to visually study the appearance characteristics of plants, some organs, and roots. In this field, with the development of computer technology and the continuous practice of researchers, the visualization model has changed from the initial mathematical model to computer tomography, and then it upgraded to feature extraction from the image. We found from the classification of model visualization types that the type changes from static to dynamic. At the same time, we found that the purpose of visualization also advanced from visual display to the combination of plant internal mechanism and phenotypic feature changes. More in-depth research can even extract plant lesions through images and apply dynamic visualization to simulate plant appearance changes. We mainly follow the SMS method to classify papers by answering seven questions, extracting data for analysis on research types, research goals, model construction methods, visual model categories, number of publications, tools, and methods. This article hopes to provide researchers with the current status of the $3 \mathrm{D}-\mathrm{PMV}$ 
field after analyzing the research data, which can help researchers find a suitable research direction in the 3D-PMV field. In addition, we also discussed the challenges faced by $3 \mathrm{D}-\mathrm{PVM}$ and the research directions that need more attention in the future. At present, researchers in the field of 3D-PMV use many types of methods, but there are fewer iterations of the same method, and most methods focus on visually displaying the shape and structure of plants. Therefore, we believe that in the future, researchers need to pay more attention to the study of dynamic visualization methods of plant growth. At the same time, researchers must continue to deepen the exploration of existing visualization methods to ensure the sustainable development of 3D-PMV. In the future, we will continue to pay attention to the latest research in the field of 3D-PMV.

\section{Conflicts of Interest}

The authors declare that they have no conflicts of interest.

\section{Acknowledgments}

This study was supported in part by the Ningxia Key Research and Development Projects under grant no. 2018BEB04020, in part by the Young Scholar in Western China of Chinese Academy of Sciences under grant no. XAB2018AW12, and in part by the National Natural Science Foundation of China under grant no. 61862049.

\section{References}

[1] K. Petersen, R. Feldt, S. Mujtaba, and M. Mattsson, "Systematic mapping studies in software engineering," in Proceedings of the 12th International Conference on Evaluation and Assessment in Software Engineering (EASE), pp. 1-10, Italy, Europe, June 2008.

[2] K. Petersen, S. Vakkalanka, and L. Kuzniarz, "Guidelines for conducting systematic mapping studies in software engineering: an update," Information and Software Technology, vol. 64, pp. 1-18, 2015.

[3] C. Wohlin, "Guidelines for snowballing in systematic literature studies and a replication in software engineering," in Proceedings of the 18th international conference on evaluation and assessment in software engineering, pp. 1-10, London, UK, May 2014.

[4] B. Kitchenham and S. Charters, Guidelines for Performing Systematic Literature Reviews in Software engineering, EBSE Technical Report, Keele University and Durham University, Keele, England, 2007.

[5] D. Šmite, C. Wohlin, T. Gorschek, and R. Feldt, "Empirical evidence in global software engineering: a systematic review," Empirical Software Engineering, vol. 15, no. 1, pp. 91-118, 2010.

[6] J. A. García-Berná, S. Ouhbi, B. Benmouna, G. García-Mateos, J. L. Fernández-Alemán, and J. M. Molina-Martínez, "Systematic mapping study on remote sensing in agriculture," Applied Sciences, vol. 10, no. 10, p. 3456, 2020.

[7] E. Fernando, S. Assegaff, and A. H. H. Rohayani, "Trends information technology in E-agriculture: a systematic literature review," in Proceedings of the 2016 3rd International Conference on Information Technology, Computer, and
Electrical Engineering (ICITACEE), pp. 351-355, Semarang, Indonesia, October 2016.

[8] A. Zewge and Y. Dittrich, "Systematic mapping study of information technology for development in agriculture (the case of developing countries)," The Electronic Journal on Information Systems in Developing Countries, vol. 82, no. 1, pp. 1-25, 2017.

[9] X. Miao and Y. Ge, "Energy management for energy harvesting-based embedded systems: a systematic mapping study," Journal of Electrical and Computer Engineering, vol. 2020, Article ID 5801850, 2020.

[10] R. Wieringa, N. Maiden, N. Mead, and C. Rolland, "Requirements engineering paper classification and evaluation criteria: a proposal and a discussion," Requirements Engineering, vol. 11, no. 1, pp. 102-107, 2006.

[11] J. M. Jurado, L. Ortega, J. J. Cubillas, and F. R. Feito, "Multispectral mapping on 3D models and multi-temporal monitoring for individual characterization of olive trees," Remote Sensing, vol. 12, no. 7, p. 1106, 2020.

[12] X. Zhao, L. Xing, S. Shen, J. Liu, and D. Zhang, "Non-destructive 3D geometric modeling of maize root-stubble in-situ via X-ray computed tomography," International Journal of Agricultural and Biological Engineering, vol. 13, no. 3, pp. 174-179, 2020.

[13] T. Van Pham, B. C. Lee, and S. A. King, "A comparison of effectiveness between 2-dimensional and 3-dimensional data visualization in detecting plant architectural characteristics," Human Interface and the Management of Information. Visual Information and Knowledge Management, Springer International Publishing, Cham, Switzerland, pp. 223-236, 2019.

[14] X. Chen, R. He, Q. Ding, and Q. Sun, "A digitization and visualization procedure for 3D wheat root system Architecture in RiceWheat rotation," Journal of The Institution of Engineers (India): Series A, vol. 100, no. 1, 2019.

[15] F. Peng, H. Zheng, S. Lu, Z. Shi, X. Liu, and L. Li, "Growth model and visualization of a virtual jujube tree," Computers and Electronics in Agriculture, vol. 157, pp. 146-153, 2019.

[16] G. Li, T. Qian, and S. Lu, "Real-time measurement, computing and visualization of plant structure," in IOP Conference Series: Materials Science and Engineeringno. 1, Bristol, UK, IOP Publishing, 2019.

[17] H. Liu, S. Li, Y. Zhu, S. Liu, and S. Li, "Wheat growth process 3D visualization research based on growth model," in Proceedings of the 11th International Conference on Computer and Computing Technologies in Agriculture (CCTA), pp. 217-231, Jilin, China, August 2019.

[18] P. Maenhout, S. Sleutel, H. Xu, L. Van Hoorebeke, V. Cnudde, and S. De Neve, "Semi-automated segmentation and visualization of complex undisturbed root systems with X-ray $\mu \mathrm{CT}$," Soil and Tillage Research, vol. 192, pp. 59-65, 2019.

[19] W. Wen, B. Li, B.-j. Li, and X. Guo, "A leaf modeling and multi-scale remeshing method for visual computation via hierarchical parametric vein and Margin representation," Frontiers of Plant Science, vol. 9, p. 783, 2018.

[20] M. Jaeger, S. Sabatier, P. Borianne et al., "Data visualization for vegetal landscapes: building 3D representations of organ biomass compartments: how plant production could constrain 3D lollypop-like representations," in Proceedings of the 2018 6th International Symposium on Plant Growth Modeling, Simulation, Visualization and Applications (PMA), pp. 85-93, Hefei, China, November 2018.

[21] A. Tabb, K. E. Duncan, and C. N. Topp, "Segmenting root systems in X-ray computed tomography images using level sets," in Proceedings of the 2018 IEEE Winter Conference on 
Applications of Computer Vision (WACV), pp. 586-595, Lake Tahoe, NV, USA, March 2018.

[22] S. Kato, T. Narita, C. Tomiyama, T. Ijiri, and H. Tanaka, “4D computed tomography measurement for growing plant animation," in Proceedings of the SA '17: SIGGRAPH Asia 2017, Bangkok Thailand, November 2017.

[23] X. Li, X. Wang, H. Wei et al., "A technique system for the measurement, reconstruction and character extraction of rice plant architecture," PloS One, vol. 12, no. 5, Article ID e0177205, 2017.

[24] X. Jing, M. Teng, Y. Tao et al., "Method for three dimensional visualization OF plant lesion appearance," Bangladesh Journal of Botany, vol. 46, no. 3, pp. 1079-1087, 2017.

[25] A. Aijazi, P. Checchin, L. Malaterre, and L. Trassoudaine, "Automatic detection and parameter estimation of trees for forest inventory applications using 3D terrestrial LiDAR," Remote Sensing, vol. 9, no. 9, p. 946, 2017.

[26] M. Li, S. Xu, Y. Yang, L. Guo, and J. Tong, "A 3D simulation model of corn stubble cutting using finite element method," Soil and Tillage Research, vol. 166, pp. 43-51, 2017.

[27] M. Mohan, C. Silva, C. Klauberg et al., "Individual tree detection from unmanned aerial vehicle (UAV) derived canopy height model in an open canopy mixed conifer forest," Forests, vol. 8, no. 9, pp. 340-9, 2017.

[28] M. Filippucci, G. Rinchi, A. Brunori, L. Nasini, L. Regni, and P. Proietti, "Architectural modelling of an olive tree. Generative tools for the scientific visualization of morphology and radiation relationships," Ecological Informatics, vol. 36, pp. 84-93, 2016.

[29] Y.-Y. Han, B.-G. Wu, K.-Y. Wang, E.-Y. Guo, C. Dong, and Z.-B. Wang, "Individual-tree form growth models of visualization simulation for managed Larix principis-rupprechtii plantation," Computers and Electronics in Agriculture, vol. 123, pp. 341-350, 2016.

[30] X. Gu, L. Xu, D. Li, and P. Zhang, "3D reconstruction and visualization of plant leaves," in Proceedings of the Sixth International Conference on Graphic and Image Processing (ICGIP 2014), Beijing, China, October 2015.

[31] J. Behmann, A.-K. Mahlein, S. Paulus, H. Kuhlmann, E.-C. Oerke, and L. Plümer, "Generation and application of hyperspectral 3D plant models," in Lecture Notes in Computer Science (Including Subseries Lecture Notes in Artificial Intelligence and Lecture Notes in Bioinformatics), pp. 117-130, Springer Nature Switzerland, Zurich, Switzerland, 2015.

[32] J. Behmann, A.-K. Mahlein, S. Paulus, H. Kuhlmann, E.-C. Oerke, and L. Plümer, "Calibration of hyperspectral close-range pushbroom cameras for plant phenotyping," ISPRS Journal of Photogrammetry and Remote Sensing, vol. 106, pp. 172-182, 2015.

[33] J. Torres-Sánchez, F. López-Granados, N. Serrano, O. Arquero, and J. M. Peña, "High-throughput 3-D monitoring of agricultural-tree plantations with unmanned aerial vehicle (UAV) technology," PLoS One, vol. 10, no. 6, Article ID e0130479, 2015.

[34] J. Wu, L. Pagès, Q. Wu, B. Yang, and Y. Guo, "Three-dimensional architecture of axile roots of field-grown maize," Plant and Soil, vol. 387, no. 1-2, pp. 363-377, 2015.

[35] S. Paulus, H. Schumann, H. Kuhlmann, and J. Léon, "Highprecision laser scanning system for capturing 3D plant architecture and analysing growth of cereal plants," Biosystems Engineering, vol. 121, no. 1-11, 2014.

[36] P. J. Zarco-Tejada, R. Diaz-Varela, V. Angileri, and P. Loudjani, "Tree height quantification using very high resolution imagery acquired from an unmanned aerial vehicle
(UAV) and automatic 3D photo-reconstruction methods," European Journal of Agronomy, vol. 55, pp. 89-99, 2014.

[37] T. Ijiri, S. Yoshizawa, H. Yokota, and T. Igarashi, "Flower modeling via X-ray computed tomography," ACM Transactions on Graphics, vol. 33, no. 4, pp. 1-10, 2014.

[38] N. Koebernick, U. Weller, K. Huber et al., "In situ visualization and quantification of three-dimensional root system Architecture and growth using X-ray computed tomography," Vadose Zone Journal, vol. 13, no. 8, 8 pages, Article ID vzj2014.03.0024, 2014.

[39] S. Mairhofer, C. Sturrock, D. M. Wells, M. J. Bennett, S. J. Mooney, and T. P. Pridmore, "On the evaluation of methods for the recovery of plant root systems from X-ray computed tomography images," Functional Plant Biology, vol. 42, no. 5, pp. 460-470, 2015.

[40] S. Paulus, J. Behmann, A.-K. Mahlein, L. Plümer, and H. Kuhlmann, "Low-cost 3D systems: suitable tools for plant phenotyping," Sensors, vol. 14, no. 2, pp. 3001-3018, 2014.

[41] T. Kuester, D. Spengler, J.-F. Barczi, K. Segl, P. Hostert, and H. Kaufmann, "Simulation of multitemporal and hyperspectral vegetation canopy bidirectional reflectance using detailed virtual 3-D canopy models," IEEE Transactions on Geoscience and Remote Sensing, vol. 52, no. 4, pp. 2096-2108, 2014.

[42] J. Liang, A. Zia, J. Zhou, and X. Sirault, "3D plant modelling via hyperspectral imaging," in Proceedings of the 2013 IEEE International Conference on Computer Vision Workshops (ICCVW), pp. 172-177, Sydney, NSW, Australia, December 2013.

[43] Y. Li, X. Fan, N. J. Mitra, D. Chamovitz, D. Cohen-Or, and B. Chen, "Analyzing growing plants from 4D point cloud data," ACM Transactions on Graphics, vol. 32, no. 6, pp. 1-10, 2013.

[44] S. Mairhofer, S. Zappala, S. Tracy et al., "Recovering complete plant root system architectures from soil via X-ray $\mu$-Computed Tomography," Plant Methods, vol. 9, no. 1, p. 8, 2013.

[45] X. Cai, Y. Sun, Y. Zhao et al., "Smart detection of leaf wilting by $3 \mathrm{D}$ image processing and 2D Fourier transform," Computers and Electronics in Agriculture, vol. 90, pp. 68-75, 2013.

[46] S. Paulus, J. Dupuis, A.-K. Mahlein, and H. Kuhlmann, "Surface feature based classification of plant organs from 3D laserscanned point clouds for plant phenotyping," $B M C$ Bioinformatics, vol. 14, no. 1, p. 238, 2013.

[47] C. N. Topp, A. S. Iyer-Pascuzzi, J. T. Anderson et al., “3D phenotyping and quantitative trait locus mapping identify core regions of the rice genome controlling root architecture," Proceedings of the National Academy of Sciences, vol. 110, no. 18, pp. E1695-E1704, 2013.

[48] L. Tang, C. Chen, H. Huang, L. Zou, and D. Lin, “A coupled architecture and physiology 3D growth model for young longan tree," in Proceedings of the 2012 IEEE 4th International Symposium on Plant Growth Modeling, Simulation, Visualization and Applications, pp. 371-378, Shanghai, China, October 2012.

[49] L. Pagès, D. Moreau, V. Sarlikioti, H. Boukcim, and C. Nguyen, "ArchiSimple: a parsimonious model of the root system architecture," in Proceedings of the 2012 IEEE 4th International Symposium on Plant Growth Modeling, Simulation, Visualization and Applications, pp. 297-303, Shanghai, China, October 2012.

[50] C. Fournier and C. Pradal, "A plastic, dynamic and reducible 3D geometric model for simulating gramineous leaves," in Proceedings of the 2012 IEEE 4th International Symposium on 
Plant Growth Modeling, Simulation, Visualization and Applications, pp. 125-132, Shanghai, China, October 2012.

[51] C. Bellasio, J. Olejníčková, R. Tesař, D. Šebela, and L. Nedbal, "Computer reconstruction of plant growth and chlorophyll fluorescence emission in three spatial dimensions," Sensors, vol. 12, no. 1, pp. 1052-1071, 2012.

[52] G. Louarn, S. Carré, F. Boudon, A. Eprinchard, and D. Combes, "Characterization of whole plant leaf area properties using laser scanner point clouds," in Proceedings of the 2012 IEEE 4th International Symposium on Plant Growth Modeling, Simulation, Visualization and Applications, Shanghai, China, October 2012.

[53] A. Paproki, X. Sirault, S. Berry, R. Furbank, and J. Fripp, "A novel mesh processing based technique for 3D plant analysis," BMC Plant Biology, vol. 12, no. 1, p. 63, 2012.

[54] Y. Chéné, D. Rousseau, P. Lucidarme et al., "On the use of depth camera for 3D phenotyping of entire plants," Computers and Electronics in Agriculture, vol. 82, pp. 122-127, 2012.

[55] L. Xu, M. Henke, J. Zhu, W. Kurth, and G. Buck-Sorlin, “A functional-structural model of rice linking quantitative genetic information with morphological development and physiological processes," Annals of Botany, vol. 107, no. 5, pp. 817-828, 2011.

[56] W.-1. Ding, H.-j. Jin, Z.-j. Cheng, and Q. Chen, "A visualization system for tomato plant modeling," in Proceedings of the 2011 Eighth International Conference Computer Graphics, Imaging and Visualization, pp. 160-165, Singapore, August 2011.

[57] W.-L. Ding, H.-J. Jin, and Z.-F. Xu, "Realistic modeling 3D architecture of tomato plant under environmental forces and system implementation," in Proceedings of the 2011 International Conference on New Technology of Agricultural, pp. 224-227, Zibo, China, May 2011.

[58] H. W. Liu, Y. Y. Liang, and H. Zhang, "Research on the rice leaf morphological formation and its visualization," in $A d-$ vanced Materials Research, D. G. Yang, T. L. Gu, H. Y. Zhou, J. M. Zeng, and Z. Y. Jiang, Eds., Trans Tech Publications, Bäch, Switzerland, pp. 2504-2508, 2011.

[59] L. Yang, Q. Q. Zhou, Y. Wan, and H. J. He, "Three dimensional visual simulation of rice root growth," in Proceedings of the 2011 International Conference on Multimedia Technology, pp. 3289-3292, Hangzhou, China, July 2011.

[60] F. Hosoi, K. Nakabayashi, and K. Omasa, "3-D modeling of tomato canopies using a high-resolution portable scanning lidar for extracting structural information," Sensors, vol. 11, no. 2, pp. 2166-2174, 2011.

[61] B. Wagner, S. Santini, H. Ingensand, and H. Gärtner, "A tool to model 3D coarse-root development with annual resolution," Plant and Soil, vol. 346, no. 1-2, pp. 79-96, 2011.

[62] Z. Sun, S. Lu, X. Guo, and Y. Tian, "Leaf vein and contour extraction from point cloud data," in Proceedings of the 2011 International Conference on Virtual Reality and Visualization, pp. 11-16, Beijing, China, November 2011.

[63] Y. Ying Zheng, S. Gu, H. Edelsbrunner, C. Tomasi, and P. Benfey, "Detailed reconstruction of 3D plant root shape," in Proceedings of the 2011 International Conference on Computer Vision, pp. 2026-2033, Barcelona, Spain, November 2011.

[64] A. Paproki, J. Fripp, O. Salvado, X. Sirault, S. Berry, and R. Furbank, "Automated 3D segmentation and analysis of cotton plants," in Proceedings of the 2011 International Conference on Digital Image Computing: Techniques and Applications, pp. 555-560, Noosa, QLD, Australia, December 2011.
[65] S. Zhou and X. Deng, "A new visualization method for the growth of corn leaf," in Proceedings of the 2010 International Conference on Intelligent Computation Technology and Automation, pp. 254-257, Changsha, China, May 2010.

[66] W. Ding, Y. Zhang, Y. Zhu, Z. Cheng, and D. Zhu, "A visualization system for rice plant modeling," in Proceedings of the 2010 International Conference on Intelligent Control and Information Processing, pp. 393-397, Dalian, China, August 2010.

[67] S. Li and Y. Zhu, "Three-dimension visualization for primary wheat diseases based on simulation model," IFIP Advances in Information and Communication Technology, vol. 317, pp. 375-381, 2010.

[68] R. P. d. M. Frasson and W. F. Krajewski, "Three-dimensional digital model of a maize plant," Agricultural and Forest Meteorology, vol. 150, no. 3, pp. 478-488, 2010.

[69] J. Vos, J. B. Evers, G. H. Buck-Sorlin, B. Andrieu, M. Chelle, and P. H. B. de Visser, "Functional-structural plant modelling: a new versatile tool in crop science," Journal of Experimental Botany, vol. 61, no. 8, pp. 2101-2115, 2009.

[70] B. Wagner, H. Gärtner, H. Ingensand, and S. Santini, "Incorporating 2D tree-ring data in 3D laser scans of coarse-root systems," Plant and Soil, vol. 334, no. 1-2, pp. 175-187, 2010. 